\title{
Jahn-Teller effects and surface interactions in multiply-charged fullerene anions and the effect on scanning tunneling microscopy images
}

\author{
Janette L Dunn ${ }^{\mathrm{a}, *}$, Haifa S Alqannas ${ }^{\mathrm{a}, \mathrm{b}}$, Andrew J Lakin ${ }^{\mathrm{a}}$ \\ ${ }^{a}$ School of Physics 8 Astronomy, University of Nottingham, University Park, Nottingham, \\ $N G 7$ 2RD, UK \\ ${ }^{b}$ Department of Physics, Faculty of Science for Girls, King Abdulaziz University, Jeddah, \\ Saudi Arabia
}

\begin{abstract}
We investigate the combined effects of Jahn-Teller (JT) coupling and interactions with a surface substrate on fullerene anions $\mathrm{C}_{60}^{2-}$ to $\mathrm{C}_{60}^{4-}$. JT coupling alone causes the $\mathrm{C}_{60}$ ions to instantaneously distort from the icosahedral symmetry of the neutral molecule to a lower symmetry, with the molecule moving dynamically between a set of equivalent distortions. When adsorbed on a surface, the number of equivalent minimum-energy distortions is reduced. The implications of this on observed scanning tunneling microscopy (STM) images will be discussed, and comparisons made with existing experimental data. We show that a consistent interpretation of the images from all of the charge states of $\mathrm{C}_{60}$ can only be obtained using a JT model in which the symmetry is further reduced by surface interactions. The comparison with experimental data also allows us to determine relationships between the quadratic Jahn-Teller coupling and surface interaction parameters.
\end{abstract}

Keywords: Jahn-Teller, fullerene, fulleride, STM

PACS: 61.48.--c, 71.70.Ej

\footnotetext{
${ }^{*}$ Corresponding author

Email address: janette.dunn@nottingham.ac.uk (Janette L Dunn)

${ }^{1}$ (C)2015. This manuscript version is made available under the $\mathrm{CC}-\mathrm{BY}-\mathrm{NC}-\mathrm{ND} 4.0$ license http://creativecommons .org/licenses/by-nc-nd/4.0/
} 


\section{Introduction}

Fullerides of the form $\mathrm{A}_{n} \mathrm{C}_{60}$ (where $\mathrm{A}$ is an alkali metal such as $\mathrm{K}, \mathrm{Rb}$ or Cs) have been the subject of many theoretical and experimental studies. Much of this interest is because the $\mathrm{A}_{3} \mathrm{C}_{60}$ fullerides can be superconducting up to 5 relatively high temperatures, or are metallic, whereas the $\mathrm{A}_{4} \mathrm{C}_{60}$ fullerides are insulators [1, 2]. The mechanisms involved in these different behaviors is believed to be related to the Jahn-Teller (JT) effect [3, 4], where coupling between the electrons and vibrations of individual $\mathrm{C}_{60}$ ions, as well as cooperative JT interactions between fullerene ions [5, 6], results in a lowering of their symmetry

10 from the icosahedral symmetry of the neutral molecule. It has recently been suggested that a similar mechanism could result in room-temperature superconductivity of substitutionally-doped graphene [7]. The insulating nature of $\mathrm{A}_{4} \mathrm{C}_{60}$ can be described by a combination of JT effects and a Mott-Hubbard band picture. This mechanism was discussed in principle as an extension of a 15 nonmagnetic model for Mott insulators with an $E \otimes e$ JT effect [8], and numerical diagonalization of the JT matrix resulted in the low-spin ground state that is required for the interpretation to hold [9]. It is therefore important to have a good understanding of the JT effect in fullerene ions. However, there is currently conflicting information on both the symmetry and the strength of the JT distortion, as well as whether the JT effect is dynamic or static.

The effect of $\mathrm{JT}$ coupling on an isolated $\mathrm{C}_{60}$ ion is to produce a number of equivalent minimum-energy distortions, each of which is characterized by a well in the adiabatic potential energy surface (APES). If the barrier between the wells is large, the system is unable to convert from one minimum-energy 25 distortion to another and the system becomes locked in one distortion, in what is known as a static JT effect. However, if the barrier is small enough to allow the system to tunnel from one well to another, the JT effect will be dynamic. The quantum-mechanical states of the system are linear combinations of products of electronic and vibrational states. The dynamical result is often interpreted 30 in terms of a hindered pseudorotation between equivalent distortions (i.e. a 
rotation of the distortion rather than a rotation of the molecule as a whole, which would typically require a much higher temperature) [10], in which the system would exhibit an instantaneous distortion if a snapshot could be taken at a given instant. However, the dynamical states do not represent a distorted 35 structure. Experimental measurements on a system subject to a dynamic JT effect could capture a specific minimum-energy distortion if the measurements are made on a timescale that is faster than the time taken to pseudorotate between equivalent distortions. However, given that pseudorotation is likely to progress on a femtosecond timescale [11], experimental measurements are 40 usually too slow for this to occur.

Factors such as interactions with neighboring fullerene ions or with a surface substrate can remove the equivalencies between some of the minimum-energy distortions that occur with isolated ions. If the result is a single minimumenergy configuration, the system will exhibit a static distortion. If there are

45 more than one equivalent minima, then the system will still perform a hindered pseudorotation but between a subset of the the minimum-energy distortions of the isolated ion.

A clear example of JT dynamics can be seen in near-infrared spectra and neutron diffraction measurements on bulk $\mathrm{A}_{4} \mathrm{C}_{60}$ salts [3]. At low temperatures, ${ }_{50}$ the $\mathrm{C}_{60}^{4-}$ ions in $\mathrm{K}, \mathrm{Rb}$ and $\mathrm{Cs}$ salts all show a $D_{2 h}$ distortion. This can be interpreted in terms of a static distortion due to the combination of JT effects and intermolecular interactions. At higher temperatures, the intermolecular interactions are no longer strong enough to stabilize a static distortion in the $\mathrm{K}$ and $\mathrm{Rb}$ salts. Individual ions become decoupled from the lattice and show $D_{5 d}$

55 or $D_{3 d}$ molecular point group symmetry [3]. Fundamental symmetry considerations involving the epikernel principle also suggest that, from a theoretical point of view, the symmetry should be $D_{3 d}$ or $D_{5 d}$ [12]. Analytical considerations of the JT effect that apply to isolated $\mathrm{C}_{60}^{4-}$ ions (as well as $\mathrm{C}_{60}^{2-}$ ) indicate that distortions are most likely to be of $D_{5 d}$ or $D_{3 d}$ symmetry, although the results

60 do depend on the values of the quadratic JT coupling and the Coulomb term splitting between different terms, which must also be taken into account [13]. 
$D_{2 h}$ symmetry only arises for a relatively small range of quadratic coupling [14]. However, there are ranges of parameters for which the three types of symmetry are very similar in energy, as shown in Fig. 2 of Ref. [14]. Evidence for a JT distortion has been seen in neutron and X-ray powder diffraction experiments on $\mathrm{Cs}_{4} \mathrm{C}_{60}$, but no discussion was given of the possible symmetry of the distortion [15].

Despite the above evidence suggesting that $D_{5 d}$ or $D_{3 d}$ JT distortions are most likely for isolated $\mathrm{C}_{60}^{4-}$ ions, calculations using density functional theory 70 (DFT) in which isolated $\mathrm{C}_{60}^{4-}$ ions were given small initial distortions of $D_{5 d}$, $D_{3 d}$ and $D_{2 h}$ symmetry and then allowed to relax to minimize the energy found that all three geometries have essentially the same energy [16]. Comparison of the resultant simulated scanning tunneling microscopy (STM) images with experimental images of a $\mathrm{C}_{60}$ monolayer intercalated with $\mathrm{K}$ on a $\mathrm{Au}(111)$ sur-

75 face led the authors to conclude that the $\mathrm{C}_{60}^{4-}$ ions must be subject to a static distortion to $D_{2 h}$ symmetry. Their model is that the transition from metallic to insulating behavior as the charge state changes from 3 to 4 is caused by a strong static JT effect in $\mathrm{C}_{60}^{4-}$ ions. However, as the experimental images in Ref. [16] that were interpreted in terms of a $D_{2 h} \mathrm{JT}$ distortion involve $\mathrm{C}_{60}^{4-}$ ions in a so monolayer, they are subject to interactions with both neighboring ions and the surface substrate. These interactions were not included in the DFT calculations. As well as the difference in the symmetry of the proposed JT distortion to that expected from the other evidence, we would not expect STM measurements to record static JT distortions of isolated molecules given that STM scans of a $\mathrm{C}_{60}$

85 molecule take the order of minutes. We note that care must be taken when performing DFT calculations that include electron-phonon coupling effects as the results obtained can depend on the functional used, as has been discussed for $\mathrm{C}_{60}$ in particular [17], although this does not necessarily mean that the DFT results presented for $\mathrm{C}_{60}^{4-}$ are incorrect.

Similar discrepancies in the suggested symmetry of the distortion apply to the monoanion. An analysis of the electronic spectra of $\mathrm{C}_{60}^{-}$ions in solution can only be interpreted in terms of a $D_{3 d}$ distortion [18], as were gas phase 
spectra of $\mathrm{C}_{60}^{-}$ions in a storage ring [19]. This is consistent with early semiempirical molecular orbital calculations that predict $D_{3 d}$ symmetry for $\mathrm{C}_{60}^{-}[20]$. Also, STM images of $\mathrm{C}_{60}$ molecules on an alkylthiol self-assembled monolayer 21] show evidence that the intrinsic JT effect would cause a $D_{3 d}$ distortion, with a further reduction in symmetry due to interactions with the surface substrate 22]. On the other hand, ab initio Hartree-Fock calculations suggest that the energies of $D_{5 d}, D_{3 d}$ and $D_{2 h}$ structures are very similar [23]. As with $\mathrm{C}_{60}^{4-}$, DFT calculations suggest that $D_{2 h}$ distortions in $\mathrm{C}_{60}^{-}$are also the most energetically-favorable in this charge state, although the energies of all three symmetry distortions are again found to be fairly similar 24 ]. Here, as for $\mathrm{C}_{60}^{4-}$, analytical calculations suggest distortion to $D_{5 d}$ or $D_{3 d}$ symmetry is most likely [25, 26, 27], but with $D_{2 h}$ symmetry still a possibility [28]. $\mathrm{K}_{5} \mathrm{C}_{60}$ monolayers, where the $\mathrm{JT}$ effects in $\mathrm{C}_{60}^{5-}$ ions are the same as in $\mathrm{C}_{60}^{-}$, are found to retain a JT-insulating state but with a different orientational ordering to in $\mathrm{K}_{4} \mathrm{C}_{60}$ [29]. However, no discussion was given of the symmetry of the distortion, so this does not add any additional information to our discussion. Static to dynamic JT transitions have also been reported in $\left[\mathrm{Cs}(\mathrm{THF})_{4}\right] \mathrm{C}_{60}$ crystals, which contain $\mathrm{C}_{60}^{-}$ions [30]. Also, the vibronic coupling constants have been calculated using photoelectron spectra and DFT, but the symmetry of the distortion was not discussed [31].

Discrepancies in interpretations of the nature of the JT effect also exist for $\mathrm{C}_{60}^{3-}$, although here the issue relates to the strength of the JT effect rather 115 than the symmetry of the distortion. The temperature dependence of infrared spectra in $\mathrm{Cs}_{3} \mathrm{C}_{60}$ shows evidence for the dynamic (pseudo) JT effect in $\mathrm{C}_{60}^{3-}$ ions being the origin of a dramatic change in electronic structure in the transition from a metallic state to a localized one, with the JT effect being sufficiently strong to overcome Hund's rule ordering and result in a low-spin ground state [4]. It is suggested that the symmetry reduction could be to $D_{2 h}$ as this is the most symmetric model for a bimodal distortion. Also, JT distortions in $\mathrm{C}_{60}^{3-}$ were proposed as a possible mechanism for the symmetry-lowering seen in NMR studies of bulk $\mathrm{Rb}_{3} \mathrm{C}_{60}$ material [32]. However, JT effects in a $\mathrm{K}_{3} \mathrm{C}_{60}$ monolayer 
on $\mathrm{Au}(111)$ were said to be weak [16]. The symmetry of JT distortions in isolated anions could be different for different charge states due to the additional effect of Coulomb interactions. For $\mathrm{C}_{60}^{3-}$, analytical considerations suggest that the symmetry of isolated ions should be $D_{2 h}$ or $\mathrm{C}_{2 h}$ [33]. However, we would expect the JT coupling constants that produce the distortion to be of similar strengths for all charge states, as vibronic coupling itself does not depend on the number of electrons present. Therefore, it would be surprising if the intrinsic JT effect was weak in $\mathrm{C}_{60}^{3-}$ but strong in $\mathrm{C}_{60}^{4-}$. It is possible that interactions with the surface in the monolayer effectively cancel out the strong JT effects. However, differences in the STM images of between one and three layers of $\mathrm{K}_{n} \mathrm{C}_{60}$ were explained in terms of different electron screening effects, assuming that the JT effects remain unaltered as the number of layers increases [34]. This leads to the assumption that JT effects would still be expected to be weak in bulk $\mathrm{K}_{3} \mathrm{C}_{60}$ fulleride salts, which is in contrast to what is seen in $\mathrm{Cs}_{3} \mathrm{C}_{60}$ salts.

In this paper, we present a theoretical model for all fullerene anions $\mathrm{C}_{60}^{n-}$ that takes into account JT effects in $\mathrm{C}_{60}$ ions, Coulomb (electron-electron) interactions and interactions with a surface substrate. After discussing the model in general terms, we will explore whether the STM measurements on $\mathrm{C}_{60}^{4-}$ ions can be explained by a model in which JT distortions that would be of $D_{3 d}$ or $D_{5 d}$ symmetry for isolated ions are converted to $D_{2 h}$ (or even to the lower $C_{2 h}$ symmetry) due to the surface interactions, as happens with the interactions with neighbors in fulleride salts. We will also discuss whether the experimental results for all charge states can be interpreted using consistent values for the strength of the vibronic coupling across all charge states. We will elucidate the role of quadratic vibronic coupling terms, as even though the changes in energy they cause might be small compared to the energy due to linear coupling, the effect on the dynamics is much greater, converting free pseudorotation to hindered pseudorotation.

It should be noted that our approach is intrinsically a multi-electron one. Many-body effects are often neglected when interpreting STM images, although it has recently been shown that they need to be included to interpret the tun- 
155 neling spectra of cobalt phthalocyanine molecules [35].

\section{Jahn-Teller and Coulomb effects}

All of the $\mathrm{C}_{60}^{n-}$ anions ( $n=1$ to 5 ) have partially-filled degenerate orbitals, so will be subject to JT distortions due to coupling between electronic and vibrational motion. As the problem involves coupling between a triplet orbital state and five-fold vibrations, the JT problems are usually known as $p^{n} \otimes h$ [26, 27]. $p^{1} \otimes h$ and $p^{5} \otimes h$ involve one electron or one hole respectively, so are described by the same JT Hamiltonian. This system is also known as $T \otimes h$. Similarly, $p^{2} \otimes h$ (with two electrons in the triplet state) and $p^{4} \otimes h$ (with two holes) are described by the same JT Hamiltonian as each other.

JT effects in systems with more than one electron have usually been treated from the point of view of electronic terms as this makes it easier to incorporate the effects of Coulomb interactions [26, 27]. For $\mathrm{C}_{60}^{2-}$ and $\mathrm{C}_{60}^{4-}$, there is a highspin configuration ${ }^{3} P$ (transforming as $T_{1 g}$ ) and low-spin configurations ${ }^{1} S$ and ${ }^{1} D$ (transforming as $A_{g}$ and $H_{g}$ respectively). The Hamiltonian for the high170 spin case is the same as for $n=1$, as it is simply a $T \otimes h \mathrm{JT}$ effect. For the low-spin cases, there is non-zero JT coupling within the ${ }^{1} D$ states and nonzero pseudo JT coupling between ${ }^{1} S$ and ${ }^{1} D$. Explicit forms for the linear and quadratic JT Hamiltonians have been written down taking the components of the $H$ state to transform as $d$-orbitals, and with vibrational coordinates ${ }_{175}\left\{Q_{\theta^{d}}, Q_{\epsilon^{d}}, Q_{4}, Q_{5}, Q_{6}\right\}$ that also transform in the same way as $d$-orbitals, namely $Q_{\theta^{d}} \sim d_{3 z^{2}-r^{2}}, Q_{\epsilon^{d}} \sim d_{x^{2}-y^{2}}, Q_{4} \sim d_{y z}, Q_{5} \sim d_{z x}$ and $Q_{6} \sim d_{x y}[26$, 27]. Alternatively, the Hamiltonian can be written down in the basis $Q_{\gamma}$ where $\gamma=$ $\{\theta, \epsilon, 4,5,6\}$, that has been used to construct Clebsch-Gordon coefficients [36], in which

$$
\begin{aligned}
Q_{\theta} & \sim \sqrt{\frac{3}{8}} d_{3 z^{2}-r^{2}}+\sqrt{\frac{5}{8}} d_{x^{2}-y^{2}} \\
Q_{\epsilon} & \sim \sqrt{\frac{3}{8}} d_{x^{2}-y^{2}}-\sqrt{\frac{5}{8}} d_{3 z^{2}-r^{2}} .
\end{aligned}
$$


This yields an alternative form for the JT Hamiltonian [13, 37], which is what we will use in this paper.

For $p^{3} \otimes h$, there is a high-spin configuration ${ }^{4} S$ and low-spin configurations ${ }^{2} P$ and ${ }^{2} D$ (transforming as $T_{1 u}$ and $H_{u}$ respectively). The ${ }^{4} S$ state is a singlet, so not subject to JT effects. There is no JT coupling within either the ${ }^{2} P$ or ${ }^{2} D$ states (because the reduced matrix elements are all zero, as is always the case for a half-filled shell), but there is pseudo-JT coupling between them [26, 27]. As for $p^{2} \otimes h$ and $p^{4} \otimes h$, the JT Hamiltonian can be written down in terms of a basis for the $H$ states using either $\left\{\theta^{d}, \epsilon^{d}\right\}$ [26, 27] or $\{\theta, \epsilon\}$ [36], with the latter being adopted in this paper.

In all of the charge states, the JT Hamiltonian can be written in the form [14]

$$
\mathcal{H}_{\mathrm{JT}}=V_{1} \mathcal{H}_{1}+V_{2} \mathcal{H}_{2}+V_{3} \mathcal{H}_{3}+\mathcal{H}_{\text {vib }}
$$

where $\mathcal{H}_{1}$ describes interactions that are linear in the $Q_{\gamma}$, and $\mathcal{H}_{2}$ and $\mathcal{H}_{3}$ describe interactions that are quadratic in the $Q_{\gamma} . \mathcal{H}_{\text {vib }}$ is the vibrational Hamiltonian, which when we are interested in the APES only is the harmonic oscillator potential $\sum_{\gamma} \mu \omega^{2} Q_{\gamma}^{2} / 2$, where $\mu$ is the nuclear mass and $\omega$ is the frequency of the $h_{g}$ mode under consideration. $V_{1}, V_{2}$ and $V_{3}$ can be defined consistently across the charge states [14]. It is convenient to write $V_{2}=\mu \omega^{2} V_{2}^{\prime}$ and $V_{3}=\mu \omega^{2} V_{3}^{\prime}$, as $V_{2}^{\prime}$ and $V_{3}^{\prime}$ are then dimensionless measures of the effect of the quadratic coupling.

It is a simple matter to include the effect of Coulomb interactions in all of the above systems by placing the different terms at different energies to each other. We place the energy of the singlet state in the $p^{2}$ and $p^{4}$ cases at an energy $\delta_{2}$ relative to the $H$ state, and the energy of the triplet in the $p^{3}$ case at an energy $\delta_{3}$ relative to the $H$ state [26, 27]. The $\delta_{i}$ can be positive or negative so that the $H$ state can be either lowest or highest in energy. As in Ref. [14], we then write $\delta_{i}=\left(V_{1}^{2} /\left(\mu \omega^{2}\right)\right) \delta_{i}^{\prime}$, where the $\delta_{i}^{\prime}$ are dimensionless measures of the strength of the Coulomb interactions relative to the linear JT interactions. 
Whilst we take the $\delta_{i}^{\prime}$ to be parameters of our model, estimates of the values that are expected to apply to $\mathrm{C}_{60}$ ions can be obtained using information from other sources. Firstly, we assume that the JT coupling can be modeled in terms of a single effective $h_{g}$ mode (rather than as eight separate $h_{g}$ modes) [38]. We can then estimate a value for $V_{1}^{2} /\left(\mu \omega^{2}\right)$ in $\mathrm{C}_{60}^{-}$from the JT energy $E_{\text {JT }}$ knowing that $E_{\mathrm{JT}}=V_{1}^{2} /\left(5 \mu \omega^{2}\right)$ in linear coupling [25]. In Ref. [18], visible and nearinfrared (NIR) spectra were used to deduce that the total JT energy for this 215 ion is around $57.9 \mathrm{meV}$. Their analysis of photoemission spectroscopy (PES) data 39] also indicates excellent agreement with this value. The fit obtained by the authors of Ref. [39] results in a larger estimate of $E_{\text {JT }}$ of $88.2 \mathrm{meV}$, but their analysis probably includes signal intensity not related to the $h_{a}$ modes. Ref. 31] tabulates values of $E_{\mathrm{JT}}$ derived from the PES data in Ref. [40], with their values ranging from $57.7 \mathrm{meV}$ to $65.0 \mathrm{meV}$. They also analyze the DFT data in Ref. 39] and conclude that the values are unreliable. They then calculate $E_{\mathrm{JT}}$ from DFT at three levels of approximation, obtaining values between 48.4 $\mathrm{meV}$ and $54.5 \mathrm{meV}$. They also summarize the results of previous calculations [41, 42, 43, 44, 45, 46] which suggest values ranging from $38.2 \mathrm{meV}$ to 51.0 $\mathrm{meV}$. Taken together, the above results (neglecting the probable over-estimate from Ref. 39]) suggest that $V_{1}^{2} /\left(\mu \omega^{2}\right)$ is between $0.19 \mathrm{eV}$ and $0.325 \mathrm{eV}$, with a value around $0.29 \mathrm{eV}$ appearing most likely. As $V_{1}$ has been defined consistently between the different charge states, this range will apply to all of the systems under consideration here.

The above estimates neglect the (unknown) contributions from quadratic coupling. These effects have been said to be small [47, 48], although recent analysis of the temperature dependence of the electron spin relaxation rates of $\mathrm{C}_{60}^{-}$estimates the pseudorotation barrier to be about $74 \mathrm{~cm}^{-1}$ [49]. If the $D_{3 d}$ points are minima then the $D_{2 h}$ points will be the saddle points that provide the pseudorotation barrier. Setting the difference in energy between the $D_{3 d}$ and $D_{2 h}$ points 25] to $74 \mathrm{~cm}^{-1}$ and taking $V_{1}^{2} /\left(\mu \omega^{2}\right)$ in the range suggested above suggests that $V_{3}^{\prime}$ is between $\approx 0.3$ and 0.5 , with all values of $V_{2}^{\prime}$ that result in stable JT distortions (namely $\approx-0.8$ to 0.44 14]) being possible. Even 
if quadratic coupling doesn't have a large effect on the value of the minimum energy, the change in symmetry that it induces could still have important consequences for the JT dynamics and hence the images that will be observed in STM, for example.

For $\mathrm{C}_{60}^{2-}$, Ref. 50] estimated the energy difference between the $A_{g}$ and $H_{g}$ terms using various models (including data taken from Ref. [51]) suggests that $\delta_{2}$ is around $0.29 \mathrm{eV}$ from three models, $0.230 \mathrm{eV}$ from one model and 0.673 $\mathrm{eV}$ from another model. Ref. [52] gives values which lead to $\delta_{2}=0.09 \mathrm{eV}$ from modified neglect of differential overlap (MNDO) calculations or $0.33 \mathrm{eV}$ from corrected self-consistent field (SCF) calculations. Taken together with the likely ranges for $V_{1}^{2} /\left(\mu \omega^{2}\right)$, the above results suggest that $\delta_{2}^{\prime} \approx 1$, although the value could be anywhere between $\approx 0.3$ and 3.5 .

For $\mathrm{C}_{60}^{3-}$, Ref. [50] estimated the energy difference $\delta_{3}$ between the $T_{1 u}$ and $H_{u}$ terms to be around $0.193 \mathrm{eV}$ from three models, $0.154 \mathrm{eV}$ from one model and $0.49 \mathrm{eV}$ from another model. The data in Ref. [52] (together with Table (3.71) in Ref. [26]) leads to $\delta_{3}=0.06 \mathrm{eV}$ from the MNDO results or $0.22 \mathrm{eV}$ 255 from the SCF calculations. This suggests that $\delta_{3}^{\prime} \approx 0.6$, although the value could be anywhere between $\approx 0.2$ and 2.6 .

Solutions to the total Hamiltonian (including JT contributions and the term splitting) have been analyzed previously, so only the results will be presented here. Linear coupling results in a continuous trough of equivalent-energy minima in the APES for all charge states [26, 27], but when quadratic couplings are also taken into account there are a finite number of minimum-energy points. Depending upon the values of quadratic coupling, there can be six $D_{5 d}$ minima, ten $D_{3 d}$ minima or $15 D_{2 h}$ minima for $p^{2} \otimes h$ and $p^{4} \otimes h$ (with a very small region with $30 C_{2 h}$ minima) [13], unless the $A_{g}$ state is sufficiently lower in 265 energy than the $H_{g}$ state, in which case JT effects can be suppressed [14]. For $p^{3} \otimes h$, the minima are of $C_{2 h}$ symmetry for most couplings, with $D_{2 h}$ minima occurring for a small range of couplings [33], unless either the $T_{1 u}$ state or the $H_{u}$ state is sufficiently low in energy in which case JT effects can be suppressed (as there are no couplings within the $T_{1 u}$ or $H_{u}$ states) [14]. However, this 
270 requires $\delta_{3}^{\prime} \geq 2.4$. As this is almost the upper limit of our estimate, we do not expected JT effects to be suppressed in $\mathrm{C}_{60}^{3-}$ by the Coulomb interaction.

\section{Interaction with a surface substrate}

When a $\mathrm{C}_{60}$ molecule interacts with a surface substrate, the symmetry will be lowered. We will treat JT effects to be the dominant interaction and the effect of the surface interaction as a perturbing distortion whose principal effect is to split the electronic orbitals, ignoring any effect of splitting of the vibrational mode. This is the usual approach taken when describing the effect of symmetrylowering distortions in JT systems, such as the effect of uniaxial strain on JT centres in crystals. The effect of the symmetry-lowering of the electronic orbitals is to remove the equivalence between some of the JT minima, with a smaller subset of the minima remaining lowest in energy. This effect has previously been investigated for $\mathrm{C}_{60}^{-}[22]$. We will now review how the effect of the interaction with the surface can be formulated for this case, before showing how the results can be extended to the other charge states.

285 For each charge state, we will consider cases where the molecule is adsorbed in the high-symmetry orientations of a pentagon down, hexagon down or doublebond down. Obviously, other orientations are possible. However, these do appear to be the main orientations observed experimentally [28, 53, 54].

\subsection{Monoanion, $\mathrm{C}_{60}^{-}$}

For surfaces such as $\mathrm{Ag}$ and $\mathrm{Au}$, it is appropriate to treat the surface as a material that is homogeneous in the plane of the surface. We can then assume that the effect on a $\mathrm{C}_{60}^{-}$ion of the interaction with the surface is to perturb the atoms closest to the surface in some way such that the axis perpendicular to the surface is not equivalent to two axes in the plane of the surface [22, 55]. The symmetry is reduced to $C_{5 v}, C_{3 v}$ and $C_{2 v}$ for adsorption with pentagon down, hexagon down or double-bond down respectively. We define single-electron states $\left\{\psi_{x}, \psi_{y}, \psi_{z}\right\}$ transforming as $\left\{T_{1 u x}, T_{1 u y}, T_{1 u z}\right\}$, where the 
label $z$ refers to a $C_{3}, C_{5}$ or $C_{2} z$-axis respectively. We can then use group theory to determine how the triplet splits in the new reduced symmetry. If the $\mathrm{C}_{60}$ is adsorbed with a hexagon or pentagon down, the result is that the triplet splits into a doublet $\left\{\psi_{x}, \psi_{y}\right\}$ and a singlet $\psi_{z}$, and for adsorption with a double-bond down $\psi_{x}, \psi_{y}$ and $\psi_{z}$ form singlets. It is a simple matter to write down a phenomenological form for a Hamiltonian to represent this splitting in the above basis as $[22,55]$

$$
\mathcal{H}_{s}^{(1)}=\frac{V_{1}^{2}}{\mu \omega^{2}}\left(\begin{array}{ccc}
0 & 0 & 0 \\
0 & -\Delta_{2} & 0 \\
0 & 0 & -\Delta_{1}
\end{array}\right)
$$

where $\Delta_{1}$ and $\Delta_{2}$ are dimensionless measures of the strength of the surface interaction relative to the linear JT coupling. $\Delta_{1}$ and $\Delta_{2}$ can be positive or negative, allowing for all possible orderings of the three states $\psi_{x}, \psi_{y}$ and $\psi_{z}$, and $\Delta_{2}=0$ for adsorption in the pentagon or hexagon-down orientations.

The JT Hamiltonian is usually expressed in terms of a 2-fold $z$-axis [25]. In order to consider JT and surface interaction effects together, it is necessary to write both Hamiltonians in the same basis. A matrix to convert from a $C_{2}$ $z$-axis to a $C_{5}$ or $C_{3} z$-axis by performing a rotation in the $x-z$ plane is

$$
U_{T}=\left(\begin{array}{ccc}
\cos \theta & 0 & -\sin \theta \\
0 & 1 & 0 \\
\sin \theta & 0 & \cos \theta
\end{array}\right)
$$

where $\theta=\tan ^{-1}\left(\phi^{-1}\right)$ for the $C_{5}$ case and $\tan ^{-1}\left((2+\sqrt{5}) \phi^{-1}\right)$ for the $C_{3}$ case, and where $\phi=\frac{1}{2}(1+\sqrt{5})$ is the Golden Ratio. Therefore the surface interaction 315 Hamiltonian in the $C_{2}$ basis is $U_{T}^{T} \mathcal{H}_{s}^{(1)} U_{T}$ for both these orientations.

\section{2. $\mathrm{C}_{60}^{2-}$ and $\mathrm{C}_{60}^{4-}$}

As the JT Hamiltonian for higher charge states has been specified using terms (rather than electron configurations) 26, 27], we need to formulate the surface interaction Hamiltonian in this basis also. The splitting of the different 
320 multiplets due to the symmetry reduction can be determined using group theory. We define the components of the $H_{g}$ state to be $\left\{H_{g \theta^{d}}, H_{g \epsilon^{d}}, H_{g 4}, H_{g 5}, H_{g 6}\right\}$ that transform in the same way as $d$-orbitals $\left\{d_{3 z^{2}-r^{2}}, d_{x^{2}-y^{2}}, d_{y z}, d_{z x}, d_{x y}\right\}$ respectively, where $z$ is perpendicular to the surface. For the pentagon-down case, the surface splitting then leads to $A_{g} \rightarrow A_{2}, H_{g \theta^{d}} \rightarrow A_{2},\left\{H_{g \epsilon^{d}}, H_{6}\right\} \rightarrow E_{2}$, and $\left\{H_{g 4}, H_{g 5}\right\} \rightarrow E_{1}$. Because there are two states that transform as $A_{2}$ (coming from $A_{g}$ and $H_{g \theta^{d}}$ ), it is not possible for write down a unique form for a surface interaction Hamiltonian from group theory alone as we do not know what linear combinations of the two states we should take. For the hexagon-down case, $H_{g} \rightarrow A_{2}+2 E$, so there are again repeated representations that mean we can't write down a unique form for the surface interaction. Furthermore, the split states need to be placed at different energies [55]. The net result is that there are too many unknowns in the Hamiltonian for it to be of any practical use.

An alternative approach is to work from states constructed from singleelectron states $\left\{\psi_{x}^{(i)}, \psi_{y}^{(i)}, \psi_{z}^{(i)}\right\}$ for electron $i=\{1,2\}$, where $z$ is again defined to 335 be perpendicular to the surface. The required results can be obtained using the tables in Fowler and Ceulemans [36]. The two-electron ${ }^{1} S$ and ${ }^{1} D$ basis states can be written as a product of electronic and spin parts, where the electronic parts can be written as

$$
\begin{aligned}
A_{g}^{(2)} & =-\frac{1}{\sqrt{3}}\left(\psi_{x}^{(1)} \psi_{x}^{(2)}+\psi_{y}^{(1)} \psi_{y}^{(2)}+\psi_{z}^{(1)} \psi_{z}^{(2)}\right) \\
H_{g \theta^{d}}^{(2)} & =\frac{1}{\sqrt{6}}\left(2 \psi_{z}^{(1)} \psi_{z}^{(2)}-\psi_{x}^{(1)} \psi_{x}^{(2)}-\psi_{y}^{(1)} \psi_{y}^{(2)}\right) \\
H_{g \epsilon^{d}}^{(2)} & =\frac{1}{\sqrt{2}}\left(\psi_{x}^{(1)} \psi_{x}^{(2)}-\psi_{y}^{(1)} \psi_{y}^{(2)}\right) \\
H_{g 4}^{(2)} & =\frac{1}{\sqrt{2}}\left(\psi_{z}^{(1)} \psi_{y}^{(2)}+\psi_{y}^{(1)} \psi_{z}^{(2)}\right) \\
H_{g 5}^{(2)} & =\frac{1}{\sqrt{2}}\left(\psi_{z}^{(1)} \psi_{x}^{(2)}+\psi_{x}^{(1)} \psi_{z}^{(2)}\right) \\
H_{g 6}^{(2)} & =\frac{1}{\sqrt{2}}\left(\psi_{y}^{(1)} \psi_{x}^{(2)}+\psi_{x}^{(1)} \psi_{y}^{(2)}\right) .
\end{aligned}
$$

The $A_{g}^{(2)}$ state is defined with a minus sign to give consistent phases to those 
340 used for the $p^{2} \otimes h$ JT Hamiltonian [26].

The equivalent four-electron basis states are considerably more complicated, and can't be separated into a product of electronic and spin parts. For example, the ${ }^{1} S$ state is

$$
A_{g}^{(4)}=\frac{1}{6 \sqrt{2}}[\psi(y, z)+\psi(z, x)+\psi(x, y)]
$$

where

$$
\psi(y, z)=\sum_{i, j, k, m} \psi_{y}^{(i)} \psi_{y}^{(j)} \psi_{z}^{(k)} \psi_{z}^{(m)} \varepsilon_{i j k m} \chi_{i}^{+} \chi_{j}^{-} \chi_{k}^{+} \chi_{m}^{-}
$$

where $\varepsilon_{i j k m}$ is the Levi-Civita symbol, $\chi_{i}^{ \pm}$are the spin up and down states of electron $i$ and all indices are summed from 1 to 4 . The four-electron $H_{g}$ states are complicated so are not presented here.

We can then determine the effect of the surface interaction using the Hamiltonian we have already constructed for $\mathrm{C}_{60}^{-}$; the Hamiltonian is the sum of this

350 Hamiltonian acting on electron $i$ and the same Hamiltonian acting on electron $j$. The result with respect to states $\left\{A_{g}^{(2)}, H_{g \theta^{d}}^{(2)}, H_{g \epsilon^{d}}^{(2)}, H_{g 4}^{(2)}, H_{g 5}^{(2)}, H_{g 6}^{(2)}\right\}$ with a $C_{2}, C_{5}$ or $C_{3} z$-axis can be written in block form as

$$
\mathcal{H}_{s}^{(2)}=\frac{V_{1}^{2}}{\mu \omega^{2}}\left(\begin{array}{c|c}
S_{1} & O_{T} \\
\hline O_{T} & S_{2}
\end{array}\right)
$$

where $O_{T}$ is a $3 \times 3$ empty matrix,

$$
S_{1}=\left(\begin{array}{ccc}
-\frac{2}{3}\left(\Delta_{1}+\Delta_{2}\right) & \frac{\sqrt{2}}{3}\left(2 \Delta_{1}-\Delta_{2}\right) & -\sqrt{\frac{2}{3}} \Delta_{2} \\
\frac{\sqrt{2}}{3}\left(2 \Delta_{1}-\Delta_{2}\right) & -\frac{1}{3}\left(4 \Delta_{1}+\Delta_{2}\right) & -\frac{\Delta_{2}}{\sqrt{3}} \\
-\sqrt{\frac{2}{3}} \Delta_{2} & -\frac{\Delta_{2}}{\sqrt{3}} & -\Delta_{2}
\end{array}\right)
$$

and $S_{2}$ is a diagonal $3 \times 3$ matrix with diagonal elements $\left\{-\Delta_{1}-\Delta_{2},-\Delta_{1},-\Delta_{2}\right\}$.

355 Again, $\Delta_{2}=0$ for the pentagon and hexagon-down orientations.

The form of $\mathcal{H}_{s}^{(2)}$ in Eq. (8) is a more specific version of the general form that could be written down from group theory alone. For example, for the pentagonprone orientation $A_{g}$ and $H_{g \theta^{d}}$ were both predicted to be $A_{2}$ singlets, $H_{g \epsilon^{d}}$ and $H_{g 6}$ were predicted to be one doublet, and $H_{g 4}$ and $H_{g 5}$ to be another doublet. 
360 The eigenvalues of Eq. (8) (when $\Delta_{2}=0$ ) show exactly this separation into singlets and doublets. However, from group theory alone we could not know that one doublet is at energy $\Delta_{1}$ relative to the other doublet, or know how the singlets should separate and their relative energies.

As for $\mathrm{C}_{60}^{-}$, we then need to write the surface interaction Hamiltonian with 365 a 2, 3 or 5 -fold $z$-axis in the same basis used to write down the JT Hamiltonian (or transform the JT Hamiltonian to the basis used for the surface interaction). To convert to a 2-fold $z$-axis, we can use knowledge of what happens to $x, y$ and $z$ to obtain a matrix to convert from the $C_{2}$ definition to the $C_{5}$ one which can be written in block form as

$$
U_{A H}=\left(\begin{array}{c|c}
1 & O_{A H} \\
\hline O_{A H}^{T} & U_{H}
\end{array}\right)
$$

where $O_{A H}$ is a $1 \times 5$ empty matrix and

$$
U_{H}=\left(\begin{array}{ccccc}
\frac{1}{2}\left(3 c^{2}-1\right) & \frac{\sqrt{3}}{2} s^{2} & 0 & \sqrt{3} s c & 0 \\
\frac{\sqrt{3}}{2} s^{2} & \frac{1}{2}\left(1+c^{2}\right) & 0 & -s c & 0 \\
0 & 0 & c & 0 & s \\
-\sqrt{3} s c & s c & 0 & c^{2}-s^{2} & 0 \\
0 & 0 & -s & 0 & c
\end{array}\right)
$$

where $s=\sin \theta$ and $c=\cos \theta$. We then need an additional transformation $S_{A H}$ to convert to the basis we have used to write the JT Hamiltonian using the definitions of $\theta$ and $\epsilon$ in Equation (11). This is given by

$$
S_{A H}=\left(\begin{array}{c|c}
1 & O_{A H} \\
\hline O_{A H}^{T} & S_{H}
\end{array}\right)
$$

where

$$
S_{H}=\left(\begin{array}{ccccc}
\sqrt{\frac{3}{8}} & \sqrt{\frac{5}{8}} & 0 & 0 & 0 \\
-\sqrt{\frac{5}{8}} & \sqrt{\frac{3}{8}} & 0 & 0 & 0 \\
0 & 0 & 1 & 0 & 0 \\
0 & 0 & 0 & 1 & 0 \\
0 & 0 & 0 & 0 & 1
\end{array}\right)
$$


Putting the two transformations together gives the required Hamiltonian for the surface interaction as $S_{A H} \cdot U_{A H}^{T} \cdot \mathcal{H}_{s}^{(2)} \cdot U_{A H} \cdot S_{A H}^{T}$.

\section{3. $\mathrm{C}_{60}^{3-}$}

The same procedure as above can be used to determine a form for the surface interaction for $\mathrm{C}_{60}^{3-}$ by building $T_{1 u}$ and $H_{u}$ states from sums of products of three single-electron $T_{1 u}$ states. While the states are complicated and are not separable in orbital and spin coordinates, the method results in a very simple form for the surface interaction Hamiltonian. In terms of the definitions of $\theta$ and $\epsilon$ in Equation (10), the result can be written as

$$
\mathcal{H}_{s}^{(3)}=\frac{V_{1}^{2}}{\mu \omega^{2}}\left(\begin{array}{c|c}
O_{T} & W \\
\hline W & O_{H}
\end{array}\right),
$$

after a constant of $-\Delta_{1}-\Delta_{2}$ has been removed from each diagonal element (as this only alters the energy zero), where $W$ is a diagonal matrix with diagonal elements $\left\{\Delta_{2}-\Delta_{1}, \Delta_{1},-\Delta_{2}\right\}$, and $O_{H}$ is a $5 \times 5$ empty matrix. This is a surprisingly simple result, especially as $\Delta_{2}=0$ for the pentagon and hexagonprone orientations.

The surface interaction Hamiltonian can be transformed to apply to a 2-fold $390 \quad z$-axis using

$$
U_{T H}=\left(\begin{array}{c|c}
U_{T} & O_{T H} \\
\hline O_{T H}^{T} & S_{H} \cdot U_{H} \cdot S_{H}^{T}
\end{array}\right),
$$

where $O_{T H}$ is an empty $3 \times 5$ matrix. $S_{H}$ is needed to convert $U_{H}$ to apply to the basis in Equation (11). The required Hamiltonian is therefore $U_{T H}^{T} \mathcal{H}_{s}^{(3)} U_{T H}$. Diagonalizing this shows that for the pentagon and hexagon-prone orientations, there is one quartet and two doublets. From group theory, we know that $T_{1 u}$ and $H_{u}$ split into two singlets and three doublets (some repeated). Again, the form here is a lot more specific than the general form (with accidental degeneracies) that would be obtained from group theory alone. For the double-bond prone orientation, there are eight singlets, with $A_{1}, A_{2}, B_{1}$ and $B_{2}$ all repeated twice. 


\section{Solutions of the total Hamiltonian}

$\delta_{2}^{\prime}=0$, for JT parameters that prefer a $D_{5 d}$ distortion, a single well will be lowest when $\Delta_{1}>0$ and 5 equivalent wells are lowest when $\Delta_{1}<0$ when 
Table 1: Groupings due to symmetry considerations of equivalent $D_{2 h}$ points for pentagon, hexagon and double-bond down orientations. Pairs between braces are related to each other by reflections in the $x-z$ plane.

\begin{tabular}{ll} 
Pentagon & $(A, B, C, G, H),(D, E, L, M, O),(F, I, J, K, N)$ \\
Hexagon & $(A, J, K),(B, M, I),(C, F, L),(D, E, N)$, \\
& $(G, H, O)$ \\
Double-bond & $\mathrm{A}, \mathrm{N}, \mathrm{O},(\{B, D\},\{C, E\}),(\{F, H\},\{G, I\})$ \\
& $(\{J, L\},\{K, M\})$ \\
\hline
\end{tabular}

the molecule is adsorbed in a pentagon-down orientation. For a hexagon-down orientation, three wells are lowest when $\Delta_{1}>0$ and three different wells are lowest when $\Delta_{1}<0$. For JT parameters preferring a $D_{3 d}$ distortion, there are 5 wells lowest for $\Delta_{1}>0$ and a different 5 wells lowest for $\Delta_{1}<0$ with the pentagon-down orientation. For the hexagon-down orientation, there is a single well lowest for $\Delta_{1}>0$, and 6 wells lowest for $\Delta_{1}<0$. Identification of the specific wells is given in Ref. [22]. Pseudorotation between the equivalent wells will also take place in the same manner as for $\mathrm{C}_{60}^{-}$. Non-zero values of $\delta_{2}^{\prime}=0$ alter the value of $\Delta_{1}$ at which the crossover between the different situations occurs.

For $\mathrm{C}_{60}^{3-}$, we can use symmetry to determine which wells transform into each other under operations of the icosahedral group in order to determine the groups of wells that we expect to remain degenerate when the surface interaction is introduced. However, a simpler method is to plot the $T_{1 u}$ components of the states that result in the potential minima as three-dimensional coordinates (with positive and negative phases). Points with the same magnitude of $z$ (where $z$ is the axis perpendicular to the surface) will remain degenerate as the surface interaction cannot do anything to alter their equivalence. This visual interpretation of the results has been confirmed by explicit numerical calculations.

As mentioned previously, the JT effect will produce minima of $D_{2 h}$ symme${ }_{450}$ try for some small ranges of the quadratic coupling constants $V_{2}^{\prime}$ and $V_{3}^{\prime}$, and 
minima of $C_{2 h}$ symmetry for all other values. When the $T_{1 u}$ components of the minimum-energy states are plotted on a sphere, the $D_{2 h}$ wells are found to be in the same positions as the centers of the double bonds of $\mathrm{C}_{60}$, as shown in Fig. 1. When a surface interaction is added, the minima no longer all have the same 455 energy, and their symmetry reduces to $C_{2 h}$ in all cases. We consider the surface interaction to act along one of the axes as defined in Section 3 For the wells originating from those of $D_{2 h}$ symmetry in the absence of a surface interaction, the groups of wells whose energy remains the same are found to be those given in Table 1 where the well labels are as in Fig. 1. Details of the states these 460 points represent can be found in Refs. [28] and [56]. Note that the labels were given incorrectly in Table 1 of Ref. [22].

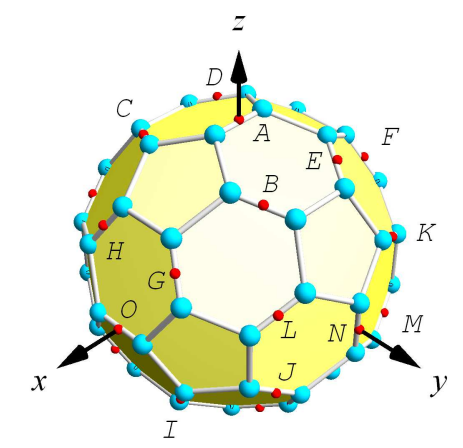

Figure 1: (Color online) Representation of the $D_{2 h}$ wells, as determined by the $T_{1 u}$ components of their electronic states. The positions are marked with dots (red online), which are equivalent to the centers of the double bonds of the $\mathrm{C}_{60}$ molecule.

Fig. 1 1 can be used to confirm the groupings found numerically. For example, wells $(A, B, C, G, H)$ are symmetrically-distributed with respect to the pentagon whose 5 -fold rotation axis is the axis in the $x-z$ plane along which the surface interaction acts. Therefore, when the diagram is re-oriented such that the axis defining the surface interaction points vertically upwards, all five of these wells are represented by points with the same vertical height. We would therefore expect these wells to remain equivalent in energy when the surface interaction is introduced, as there is nothing to alter their relative energies. The same arguments can be applied to all of the other groups of wells. The results are 
identical to those in Fig. 1 of Ref. [57] for $D_{2 h}$ wells in the $\mathrm{C}_{60}^{2+}$ cation.

For wells originating from $C_{2 h}$ wells in the absence of a surface interaction, the situation is rather more complicated than for $D_{2 h}$ as the positions of the points representing the $T_{1 u}$ components of the electronic states depend on the coupling constants. By symmetry, each of the points representing the $30 C_{2 h}$ wells must lie on one of the great circles passing through both ends of a double bond. This shows that the wells divide into the same sets for all (allowed) values of quadratic coupling. For the pentagon-down orientation, there are two sets of 10 wells and two sets of 5 . For the hexagon-down orientation, there are four sets of 6 wells and two sets of 3 wells, whereas for the double bond-down orientation, there are six sets of 4 wells and three sets of 2 wells.

Some illustrative results are given in Fig. 2 which shows the representation of two sets of wells that remain equivalent when a $\mathrm{C}_{60}$ molecule is adsorbed in a pentagon-down orientation. Changing the value of quadratic coupling moves 485 the points up and down the great circles, and points with a given magnitude of $z$ will remain degenerate when the surface interaction is introduced. Arrows on the great circles indicate a set of consistent directions in which the minima could move, although all points could equally well move in the opposite directions to the arrows. The results for the set of 5 equivalent wells shown in Fig. 2(a) 490 are relatively simple to interpret, as each well lies on a great circle passing through the center of the uppermost pentagon. The points in Fig. 2(b) are rather more complicated as the great circles involved are not in vertical planes so the points move horizontally as well as vertically as the quadratic coupling changes. Also, there are two wells on each great circle. However, the results can still be interpreted in the same way. Similar interpretations of the groupings hold for adsorption in other orientations. It should be noted that in this figure, the positions indicated are those with no surface interaction, but the groupings of equivalent wells apply when a surface interaction is introduced. 
(a)

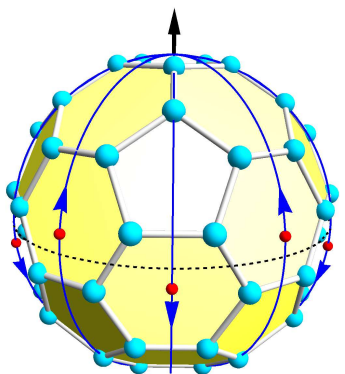

(b)

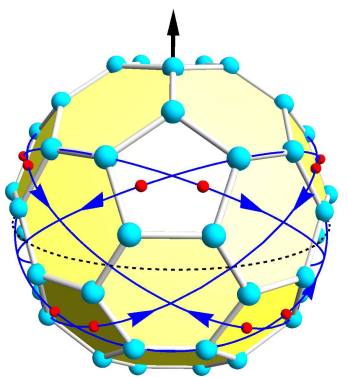

Figure 2: (Color online) Representation of the $C_{2 h}$ wells, as determined by the $T_{1 u}$ components of their electronic states. (a) and (b) show sets of 5 and 10 wells respectively that remain equivalent when a $\mathrm{C}_{60}$ molecule is adsorbed in the pentagon-down orientation. The solid curves are the great circles upon which the representations of the minima lie. The points on the great circles (red online) are the results obtained for a representative value of quadratic coupling, with the arrows on the great circles indicating consistent directions in which we could move to obtain minima for different quadratic coupling values. The dashed curve represents the equator.

\section{Simulation of STM images}

According to the standard Tersoff-Hamann interpretation [58], the current in an STM experiment with an $s$-type tip is proportional to $\sum_{\mu}\left|\psi_{\mu}\right|^{2}$, where the sum is taken over all degenerate states $\psi_{\mu}$ in a window around the energy being probed. This result arises from Bardeen's theory of tunneling from a many-particle point of view [59]. When the $\psi_{\mu}$ are multi-electron states, the theory can be extended to show that the current can be obtained from the sums of squares of multi-electron states integrated over both the spatial coordinates of all electrons except one and the spin coordinates of all electrons (i.e. the probability of finding one electron in a given volume when all other electrons can be anywhere). A similar approach is used to obtain the current in DFT approaches, although our multi-electron states arise as a result of including electron-electron and electron-vibration couplings. We find it useful to define the constant of proportionality such that $I=\sum_{\mu}\left|\psi_{\mu}\right|^{2} / \mu$, although obviously this will not give a current in physical units.

The first point to make here is that quadratic coupling has a large effect 
515 on the STM images that will be observed experimentally. We have seen that quadratic coupling results in sets of one or more equivalent minimum-energy configurations. Due to the long timescale of STM measurements, the recorded images will be a superposition of images resulting from each of the equivalent distortions [55]. If we had neglected quadratic coupling in our model, there would be a continuous trough of equivalent minimum-energy configurations. A simulated STM image would then be a superposition of states arising from all points on this trough. In general, as this is sampling a large number of configurations, we would expect predicted images to be much more like those where all components of the orbital are imaged together than those with quadratic coupling that sample many fewer configurations.

In $T \otimes h$, it has been shown that when JT effects are present, the tunneling current must be proportional to a linear combination of $\psi_{x}^{2}, \psi_{y}^{2}$ and $\psi_{z}^{2}$ for molecules adsorbed with a hexagon, pentagon or double-bond down [22] (where $\psi_{x}, \psi_{y}$ and $\psi_{z}$ are the single electron states, with the label $i$ dropped as we no longer need to refer to the different electrons). In other words, the current is

$$
I=a_{x} \psi_{x}^{2}+a_{y} \psi_{y}^{2}+a_{z} \psi_{z}^{2}
$$

where $a_{x}, a_{y}$ and $a_{z}$ are numerical coefficients. Observed STM images of $\mathrm{C}_{60}^{-}$ ions can therefore be interpreted in terms of combinations of images of $\psi_{x}^{2}$, $\psi_{y}^{2}$ and $\psi_{z}^{2}$ [22]. Due to the symmetric placement of equivalent minima, there are no 'cross terms' (such as $\psi_{x} \psi_{y}$ ) [22]. Furthermore, our chosen normalization ensures that $a_{x}+a_{y}+a_{z}=1$. As an example, consider a $\mathrm{C}_{60}^{-}$ion adsorbed in the hexagon-down orientation. If one minimum-energy well has an electronic state $c_{x} \psi_{x}+c_{y} \psi_{y}+c_{z} \psi_{z}$, then application of the $C_{3 v}$ group operations shows that the two states $c_{x}\left(-\frac{1}{2} \psi_{x} \pm \frac{\sqrt{3}}{2} \psi_{y}\right)+c_{y}\left(\mp \frac{\sqrt{3}}{2} \psi_{x}-\frac{1}{2} \psi_{y}\right)+c_{z} \psi_{z}$ must also correspond to wells with the same minimum energy. Calculating $I=\sum_{\mu}\left|\psi_{\mu}^{2}\right| / \mu$ where the sum is taken over these three states gives $I=\frac{1}{2}\left(c_{x}^{2}+c_{y}^{2}\right)\left(\psi_{x}^{2}+\psi_{y}^{2}\right)+c_{z}^{2} \psi_{z}^{2}$ 22]. Similar arguments regarding the disappearance of cross-terms hold for the pentagon-down and double bond-down orientations. Using the expressions for 
the multi-electron states in terms of single-electron states (e.g. equation (5)), the form of current in equation (16) can also be shown to hold in the multielectron cases for adsorption in the hexagon-down, pentagon-down and double bond-down orientations. Hence STM images can be interpreted in terms of combinations of images of $\psi_{x}^{2}, \psi_{y}^{2}$ and $\psi_{z}^{2}$ in these cases also.

Before proceeding, we will make some general conclusions about the form of the tunneling current just by counting the available single-electron states, without reference to any specific JT model. For the $p^{2} \otimes h$ case, it is possible to take linear combinations of the two-electron basis states $A_{g}^{(2)}, H_{g \theta^{d}}^{(2)}$ and $H_{g \epsilon^{d}}^{(2)}$ that are $\psi_{x}^{(1)} \psi_{x}^{(2)}, \psi_{y}^{(1)} \psi_{y}^{(2)}$ and $\psi_{z}^{(1)} \psi_{z}^{(2)}$. Therefore, it is possible (at least in principle) for a current to be observed for filled states that is proportional to just $\psi_{x}^{2}, \psi_{y}^{2}$ or $\psi_{z}^{2}$, subject to finding JT parameters that would give any of these results. However, for the higher charge states this is not possible. For three electrons, if the coefficient of $\psi_{x}$ (say) is zero, then we can place two electrons in $\psi_{y}$ states and one in $\psi_{z}$ or vice versa, or into combinations of $\psi_{y}$ and $\psi_{z}$ states. Therefore the coefficients of any given state can't be more than $2 / 3$. This can easily be confirmed mathematically using explicit expressions for the three-electron states. This means that it is not possible to obtain STM images that reflect purely $x, y$ or $z$ character with three electrons, so observed images will be much more generic. This mixing effect becomes even stronger for more electrons. For filled states with four electrons, if the coefficient of $\psi_{x}$ (say) is zero then two electrons would need to be in the state $\psi_{y}$ and two in $\psi_{z}$, meaning 565 the largest possible coefficient is 0.5. Again, this has been confirmed from our numerical calculations.

While the minimum-energy wells group in the same way for $\mathrm{C}_{60}^{-}, \mathrm{C}_{60}^{2-}$ and $\mathrm{C}_{60}^{4-}$, the expected STM images are different in all three cases because the electronic basis states are different. This is particularly true of images that would occur if the molecule was able to become locked in a specific distortion. However, when a set of degenerate wells is imaged together, the averaging effect of combining results due to individual wells means that the final result is much more similar in the three cases, resembling the central image in Fig. 4 of Ref. 
22] in the pentagon-down case for example.

When imaging filled states of $\mathrm{C}_{60}^{2-}$, the form of the 2-electron states suggests that it could be possible to obtain a current that is just proportional to $\psi_{x}^{2}, \psi_{y}^{2}$ or $\psi_{z}^{2}$. However, numerical investigations with our model show that the coefficients of $\psi_{x}^{2}$ and $\psi_{y}^{2}$ are always equal for the pentagon or hexagon-down orientations. Again, this is to be expected from analogy to the $T \otimes h$ case. In all cases, we find that the current is dominated by $\psi_{z}^{2}$ for positive values of $\Delta_{1}$ and by $\psi_{x}^{2}+\psi_{y}^{2}$ for negative values of $\Delta_{1}[60]$. The results are fairly independent of the precise values chosen for $\delta_{2}^{\prime}, V_{2}^{\prime}$ and $V_{3}^{\prime}$. The main dependence on these parameters is in how much the current contains a mixture of all three contributions for some small magnitudes of $\Delta_{1}$.

For filled states of $\mathrm{C}_{60}^{4-}$, we find that for the pentagon and hexagon-down orientations, the largest contribution to the current will be either $\psi_{z}^{2}$ or $\psi_{x}^{2}+\psi_{y}^{2}$, but there will be a significant contribution from the other state(s) as well [60]. For positive values of $\delta_{2}^{\prime}$, there is a crossover in which contribution is largest as $\Delta_{1}$ increases. This is because for large positive values of $\delta_{2}^{\prime}$, only the $H_{g}$ state will contribute to the results. Although the expression for $I$ is similar in both the pentagon and hexagon-down orientations, it should be remembered that the labels $x, y$ and $z$ refer to coordinates in which $z$ is perpendicular to the surface, which is different for the two cases. Hence the predicted images will be different. Results can be obtained for the double bond-down orientation in a similar way, but it is more complicated to analyze due to the additional surface interaction parameter.

For $\mathrm{C}_{60}^{3-}$, the results are dominated by $\psi_{z}^{2}$ for positive values of $\Delta_{1}$ and by $\psi_{x}^{2}+\psi_{y}^{2}$ for negative values for adsorption in both the pentagon and hexagondown orientations. The results are also found to have little dependence on the JT coupling parameters, as long as the JT coupling is sufficiently large with respect to the term splitting that JT distortions can still occur [22]. This is because the distortion with surface interactions is of $C_{2 h}$ symmetry in all cases. The limits of the coefficients when the magnitude of the surface interaction is strong can be found from the eigenvectors of the combined surface interaction 
605 and term splitting Hamiltonians, with no JT effect. This shows that the current will tend to the limits of $\left(\psi_{x}^{2}+\psi_{y}^{2}+4 \psi_{z}^{2}\right) / 6$ when $\Delta_{1}$ is positive and $\left(\psi_{x}^{2}+\psi_{y}^{2}\right) / 2$ when $\Delta_{1}$ is negative, irrespective of the value of $\delta_{3}^{\prime}$. The only difference is the rate at which the limits are attained. For the double bond-down orientation, the current can have a coefficient $\approx 2 / 3$ for $\psi_{x}^{2}, \psi_{y}^{2}$ or $\psi_{z}^{2}$, with a value of $\approx 1 / 3$ ${ }_{610}$ for one of the other contributions and 0 for the third contribution 60$]$.

\section{Comparison with experimental results}

We will now show how our theoretical results can be used to help explain experimentally-observed STM images. We have seen that for all three of the orientations we have considered, the resultant STM images arising from hopping between equivalent JT-distorted configurations can all be built from a superposition of images of $\psi_{x}^{2}, \psi_{y}^{2}$ and $\psi_{z}^{2}$, as there are no cross-terms in the square of the total wavefunction. We can therefore determine the contributions from $\psi_{x}^{2}$, $\psi_{y}^{2}$ and $\psi_{z}^{2}$ required in order to obtain the best match between our simulations and any experimental image. We can then determine what possible ranges for the JT coupling constants and surface interaction parameters can result in that match.

The majority of STM measurements involving $\mathrm{C}_{60}$ probably involve the neutral molecule, which is not JT-active (although the effective charge state is not necessarily obvious as the STM technique involves electrons tunneling between a $\mathrm{C}_{60}$ molecule and the STM tip). The results that most obviously involve $\mathrm{C}_{60}$ anions involve $\mathrm{K}_{x} \mathrm{C}_{60}$ monolayers on an $\mathrm{Au}(111)$ substrate [16], with $3 \leq x \leq 4$. This suggests that $\mathrm{C}_{60}^{3-}$ or $\mathrm{C}_{60}^{4-}$ anions are likely to be present. We will concentrate on these results below. Images obtained from $\mathrm{C}_{60}$ ions in a $\mathrm{K}_{4} \mathrm{C}_{60}$ or $\mathrm{K}_{5} \mathrm{C}_{60}$ monolayer [29] or multilayer [34] should also involve JT distortions, so we could also expect them to be described by the results in this paper. However, the images in the literature are not sufficiently distinctive for any identification to be made. 


\subsection{Match to $C_{60}^{4-}$}

The experimental image from Ref. [16] attributed to filled states of $\mathrm{C}_{60}^{4-}$ orientation. This is a rather generic image that can be obtained for a very wide range of possible currents. The result for a constant current simulation of $I=\left(\psi_{x}^{2}+\psi_{y}^{2}\right) / 2$ is shown in figure 3(a). Images of many other currents, such as $\left(\psi_{x}^{2}+\psi_{y}^{2}+\psi_{z}^{2}\right) / 3$ or even just $\psi_{x}^{2}$, also produce similar results, although in exhibit four lobes, and is much more distinctive than that of the filled states. Examining the images for $\psi_{x}^{2}, \psi_{y}^{2}$ and $\psi_{z}^{2}$ for the pentagon, hexagon and doublebond prone orientations suggests that the image arises from predominantly the $\psi_{y}^{2}$ component of the double-bond prone orientation, with a small contribution from $\psi_{x}^{2}$. This has been discussed previously, where the image for $I=0.015 \psi_{x}^{2}+$ $0.985 \psi_{y}^{2}$ was presented [55]. In fact, images that to the eye appear to closely resemble the experimental image can be obtained when $a_{y}$ is between $\approx 0.92$ and 0.985 and a small contribution from $\psi_{z}^{2}$ can also be introduced, although it should not exceed the $\psi_{x}^{2}$ contribution and is ideally less than this. The result for ${ }_{655} I=0.035 \psi_{x}^{2}+0.96 \psi_{y}^{2}+0.005 \psi_{z}^{2}$, shown in figure 3 (b), is almost identical to that in Ref. [55]. It should be noted that it is not known whether any post-processing was carried out on the experimental images in Ref. [16], nor is it known whether broadening as part of the measurement process could contribute to the observed images. Factors such as these could alter our conclusions on the detailed ranges of $\left\{a_{x}, a_{y}, a_{z}\right\}$ that we deem to match the experimental data, although we would still expect the image of empty states to be dominated by $\psi_{y}^{2}$.

The next step in the identification of the origins of the experimental images is to determine what values of the JT couplings, term splitting and surface 
(a)
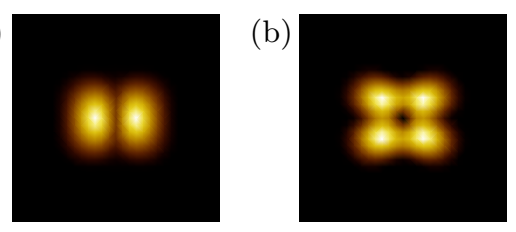

(c)

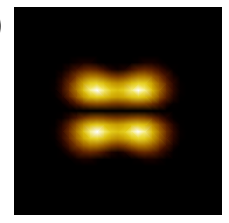

(d)

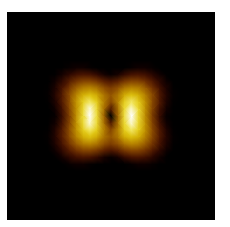

Figure 3: (Color online) Simulated STM images of double bond-down $\mathrm{C}_{60}^{4-}$ molecules. (a) $I=\left(\psi_{x}^{2}+\psi_{y}^{2}\right) / 2$, which matches experimental images of filled states, and occurs for a very wide range of JT and surface interaction parameters. (b) $I=0.035 \psi_{x}^{2}+0.96 \psi_{y}^{2}+0.005 \psi_{z}^{2}$, which matches experimental images of empty states and occurs for positive or small negative values of $\Delta_{2}$ (see text for details). (c) $I=\psi_{y}^{2}$, which arises if the JT distortion is assumed to be of $D_{2 h}$ symmetry. (d) $I=0.17 \psi_{x}^{2}+0.76 \psi_{y}^{2}+0.07 \psi_{z}^{2}$, which could be obtained with no surface interaction and JT parameters that result in a $D_{3 d}$ distortion (e.g. from $\delta_{2}^{\prime}=1$, $V_{2}^{\prime}=0$ and $V_{3}^{\prime}=0.3$ ). (c) and (d) are less like the image observed experimentally than (b).

interaction parameters could produce the required combinations of $\psi_{x}^{2}, \psi_{y}^{2}$ and ${ }_{665} \psi_{z}^{2}$. The first point to note is that the image of $\mathrm{C}_{60}^{4-}$ is of empty states, and the JT models discussed above apply to filled states. However, at least mathematically, the JT problem of empty states of $\mathrm{C}_{60}^{4-}$ must be the same as that of filled states of $\mathrm{C}_{60}^{2-}$. The $Q_{\gamma}$ will be those that minimize the energy for the $p^{4} \otimes h$ problem, but as the Hamiltonian is the same for $p^{2} \otimes h$ and $p^{4} \otimes h$, the $Q_{\gamma}$ will be the same for both problems. The images observed for $\mathrm{C}_{60}^{2-}$ and $\mathrm{C}_{60}^{4-}$ will be different however, because the current used to determine the image must be calculated using two-electron states for the empty orbitals but four-electron states for the filled orbitals.

In Ref. [16], a DFT simulation was given for the image of the empty states 16] assuming they arise due to a static JT effect of $D_{2 h}$ symmetry, without considering the effects of interactions with the surface substrate. Their DFT image has a nodal plane (with zero electron density) through the center of the image. In terms of our parameters, this result equates to a current of 
$\psi_{y}^{2}$ alone. Our image assuming this case, which is equivalent to that from the DFT calculations, is shown in Fig. 3 (c). The match to the experimental images is less good in this case. However, it is possible that factors such as experimental blurring could join the lobes in a manner that is more consistent with the experimental image, having the same effect as adding contributions from the other components. Therefore it is not possible to conclusively rule out an explanation based on a current of solely $\psi_{y}^{2}$, although this seems less likely.

Fig. 3(d) shows that when the surface interaction is neglected, the expected image changes from four lobes to two crescents. This suggests how the image would be likely to change if the $\mathrm{C}_{60}^{4-}$ ion were to be imaged on a surface where the interactions with the surface are much weaker than that on $\mathrm{Au}$.

It should also be noted that from the form of the surface interaction acting on single-electron states in Eq. (3), we can see that we can get the required match in the absence of JT effects, as long as $\Delta_{2}>0$ and $\Delta_{2}>\Delta_{1}$. In fact, these conditions can also be seen considering the form of the surface interaction in Eq. (8) directly. This is different to the $T \otimes h$ case, where results from equivalent wells had to be added together to eliminate unwanted cross-terms in the expression for the current [22]. However, to get a current of just $\psi_{y}^{2}$ requires $\delta_{2}^{\prime}=0$ (as well as the conditions on $\Delta_{1}$ and $\Delta_{2}$ ). To match a current of $0.035 \psi_{x}^{2}+0.96 \psi_{y}^{2}+0.005 \psi_{z}^{2}$ requires $\Delta_{1}=-1.06$ and $\Delta_{2}=0.77$ when $\delta_{2}^{\prime}=1$. No match is possible for $I=0.015 \psi_{x}^{2}+0.985 \psi_{y}^{2}$. It would seem fortuitous that the term splitting and surface interaction parameters happen to be related in such a way as to give the distinctive image observed experimentally, although of course this is possible. However, it is relevant to note that the ground state comes from a combination of the $A_{g}^{(2)}, H_{g \theta^{d}}^{(2)}$ and $H_{g \epsilon^{d}}^{(2)}$ basis states for all of these matches. It therefore seems appropriate to search for matches including JT and Coulomb effects that involve these three states also. For the match to be a combination of these states alone, we require $Q_{4}=Q_{5}=0$. Therefore, ground states can be sought by minimizing the lowest eigenvalue of a $3 \times 3$ matrix with respect to $Q_{\theta}, Q_{\epsilon}$ and $Q_{6}$, which is much quicker than minimizing the full $6 \times 6$ matrix with respect to the five $Q_{\gamma}$, although the same results are obtained when 
the full calculation is carried out.

We find that when quadratic coupling is included, a current dominated by $\psi_{y}^{2}$ is predicted for the same ranges of $\Delta_{1}$ and $\Delta_{2}$ as with no JT effects. However, there are now small contributions from $\psi_{x}^{2}$ and $\psi_{z}^{2}$, with the $\psi_{z}^{2}$ contribution being extremely small $\left(a_{z}<0.01\right)$ except when $\Delta_{1}$ is close to the crossover between the two different forms for the current that occurs when $\Delta_{1}=\Delta_{2}$. This meets all of the conditions we require in order to match the experimental

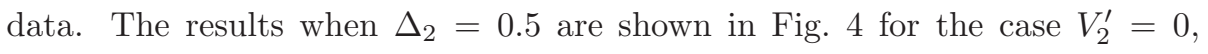
$V_{3}^{\prime}=0.1$ and $\delta_{2}^{\prime}=1$. For these parameters, the problem without the surface interaction would result in ten $D_{3 d}$ wells, but when the surface interaction is included, there is only one minimum-energy point, which has $D_{2 h}$ symmetry. The results neglecting all JT effects are also shown in the same figure. The results for other values of $V_{2}^{\prime}, V_{3}^{\prime}$ and $\delta_{2}^{\prime}$, including those that would give $D_{5 d}$ wells in the absence of any surface interactions, are very similar. The results for other values of $\Delta_{2}$ are also similar, except that the crossover position shifts to $\Delta_{1}=\Delta_{2}$.

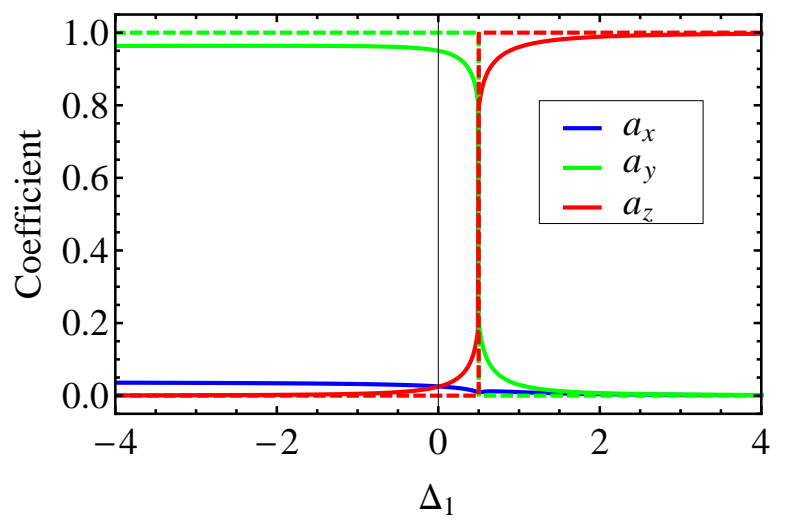

Figure 4: Variation of the coefficients $\left\{a_{x}, a_{y}, a_{z}\right\}$ in the expression for the current when $\Delta_{2}=0.5$. The solid lines are for $V_{2}^{\prime}=0, V_{3}^{\prime}=0.1$ and $\delta_{2}^{\prime}=1$, while the dashed lines are the results considering the surface interaction only.

Our match to the form of the current that we predict most closely matches the experimental image comes from positive or small negative values of $\Delta_{2}$ 
and JT parameters that would result in a $D_{3 d}, D_{5 d}$ or $D_{2 h}$ distortion without any surface interaction. The symmetry is $D_{2 h}$ in all cases when the surface interaction is included. To get a current of $\psi_{y}^{2}$ requires a larger value of $\Delta_{2}$. The predicted image in this case is the same as that obtained by Wachowiak et al. using DFT. However, our interpretation involves a JT distortion that is most likely to intrinsically be of $D_{3 d}$ or $D_{5 d}$ symmetry [14]. This is more consistent with the other experimental and theoretical results that point towards $\mathrm{C}_{60}^{-}$ions preferring a $D_{3 d}$ JT distortion [18, 19, 20, 21], although the symmetry of the JT distortion could be different in $\mathrm{C}_{60}^{4-}$ to $\mathrm{C}_{60}^{-}[14]$.

To obtain a more quantitative determination of the JT parameters that could give the required current, we need to examine the numerical results from our model. The general problem is rather complex, as the results are a function of the two quadratic JT coupling constants $V_{2}^{\prime}$ and $V_{3}^{\prime}$, the term splitting $\delta_{2}^{\prime}$, and the surface interaction parameters $\Delta_{1}$ and $\Delta_{2}$. Results have been obtained for a wide range of parameters, and all result that give states predominantly involving $A_{g}^{(2)}, H_{g \theta}^{(2)}$ and $H_{g \epsilon}^{(2)}$ are found to have $Q_{4}=0$ as well as $Q_{5}=Q_{6}=0$.

Figure[5]shows the variation in the surface interaction parameters $\Delta_{1}$ and $\Delta_{2}$ as a function of the coefficient $a_{x}$ for three different values of $a_{y}$ that will result in a match to the observed images. All curves are for zero quadratic coupling $\left(V_{2}^{\prime}=V_{3}^{\prime}=0\right)$. The solid curves are for $I=0.015 \psi_{x}^{2}+0.98 \psi_{y}^{2}+0.005 \psi_{z}^{2}$, the dashed curves are for $I=0.005 \psi_{x}^{2}+0.99 \psi_{y}^{2}+0.005 \psi_{z}^{2}$ and the dot-dashed curves are for $I=0.015 \psi_{x}^{2}+0.97 \psi_{y}^{2}+0.015 \psi_{z}^{2}$. The curves in (a) are for when a linear 750 JT effect is included, although the difference between these results and those for no JT effect are negligible (a maximum variation of \pm 0.02 ). This shows that small changes in $a_{x}$ that will not produce any visible differences in the predicted STM images will produce a big change in the value of $\Delta_{1}$ that will produce the required current. The curves in (b) are the corresponding results 755 for the variation in $\Delta_{2}$. Here, there are some differences between the JT and non-JT results, although the variation is small.

Finally, we look at the dependence of $\Delta_{1}$ and $\Delta_{2}$ on the quadratic coupling constants. To illustrate the results, we choose parameters that result in a cur- 


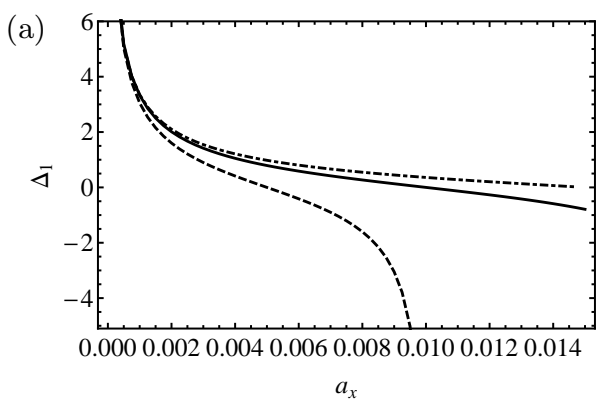

(b)

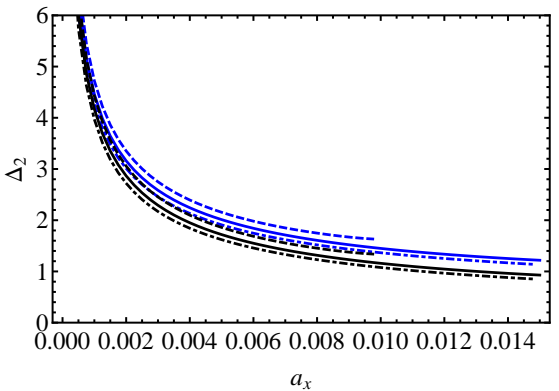

Figure 5: Variation with (a) $\Delta_{1}$ and (b) $\Delta_{2}$, of the coefficient $a_{x}$ in the expression for $I$. The solid lines are for $a_{y}=0.98$, the dashed lines for $a_{y}=0.99$ and the dot-dashed lines for $a_{y}=0.97$. In (b), the upper set of lines (blue online) is for no JT effect and the lower set (black online) is when a linear JT effect is included. Results when quadratic coupling is included are very similar to (b).

rent $I=0.015 \psi_{x}^{2}+0.98 \psi_{y}^{2}+0.005 \psi_{z}^{2}$ (i.e. a current in which the coefficients $\left\{a_{x}, a_{y}, a_{z}\right\}$ are towards the middle of what we determine to be the most likely ranges), although the results for the other currents that match the experimental data are very similar. JT effects occur in the absence of any surface interaction for all regions inside the solid outer boundary [14]. For $\delta_{2}^{\prime}=1$, the required current can be obtained for all values of $V_{2}^{\prime}$ and $V_{3}^{\prime}$ if appropriate choices are made for $\Delta_{1}$ and $\Delta_{2}$, as shown in figure 6(a) and (b). The contours show the value of $\Delta_{1}$ and of $\Delta_{2}$ required in order to produce the required current. For other values of $\delta_{2}^{\prime}$, it is not always possible to find a solution for all values of $V_{2}^{\prime}$ and $V_{3}^{\prime}$. Figure 6 (c) and (d) show that the required ground state can only be obtained when $V_{2}^{\prime}$ and $V_{3}^{\prime}$ lie within the shaded region when $\delta_{2}^{\prime}=0.3$. However, this is not expected to apply to $\mathrm{C}_{60}^{2-}$. 

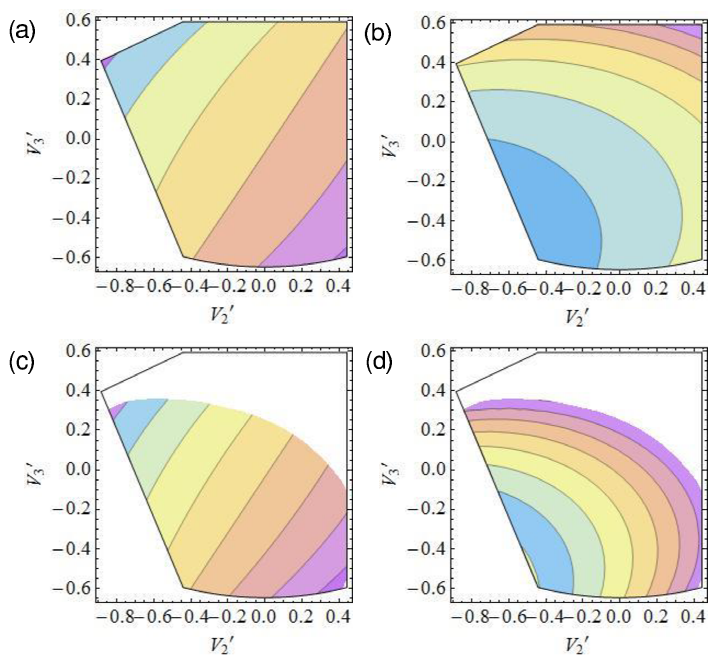

Figure 6: Dependence of $\Delta_{1}$ and $\Delta_{2}$ on the quadratic coupling coefficients $V_{2}^{\prime}$ and $V_{3}^{\prime}$ to give a current $I=0.015 \psi_{x}^{2}+0.98 \psi_{y}^{2}+0.005 \psi_{z}^{2}$. In (a) and (b), the term splitting $\delta_{2}^{\prime}=1$. (a) shows contours of $\Delta_{1}$ from -0.5 (top left) to -1 (bottom right) in steps of -0.1 . (b) shows contours of $\Delta_{2}$ from 0.5 (top right) to 1 (bottom left) in steps of 0.1. In (c) and (d), $\delta_{2}^{\prime}=0.3$, with (c) showing contours of $\Delta_{1}$ from 0 (top left) to -1.4 (bottom right) in steps of -0.2 and (d) showing $\Delta_{2}$ from 0.1 (top right) to 0.7 (bottom left) in steps of 0.1 .

\subsection{Match to $\mathrm{C}_{60}^{3-}$}

For $\mathrm{C}_{60}^{3-}$, the filled and empty states are very similar. This is to be expected because as the $p$ shell is half-full, the JT problem for filled and empty states will be the same, and also the expression for the current will be the same in both cases. It is very different to the $\mathrm{C}_{60}^{4-}$ case though, where the images of filled and empty states are very different. For both the filled and empty states, two types of images for individual molecules are seen; a dimmer 3-lobed image and a brighter triangular image [16]. Ref. [16] did not attempt to interpret these images in terms of a JT effect. The most likely explanation for the appearance of two different images is that this is due to a combination of $\mathrm{C}_{60}-\mathrm{C}_{60}$ and $\mathrm{C}_{60}$-surface interactions [61] that results in a reconstruction in which different effective values for the strength of the surface interaction are experienced by different molecules, depending on their location on the surface, and/or that the molecules are at different physical heights above the surface. Several different patterns of dim 
785 and bright $\mathrm{C}_{60}$ molecules observed on $\mathrm{Cu}(111)$ have been interpreted in terms of these mechanisms [61]. It seems reasonable to assume that differences in intermolecular detail could also occur due to these factors.

(a)

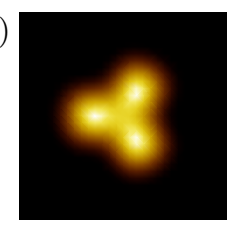

(c)

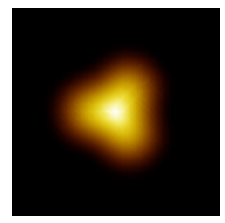

(b)

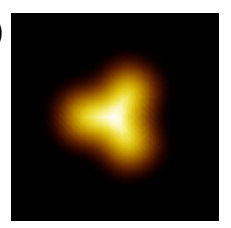

(d)

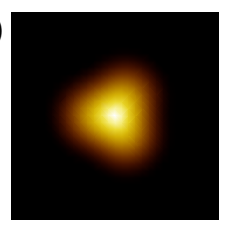

Figure 7: (Color online) Simulated STM images of hexagon-down $\mathrm{C}_{60}^{3-}$ molecules. In (a) to (d), $a_{z}=0.35,0.45,0.55$ and 0.65 respectively.

Due to their 3-fold symmetry, the observed images must arise from molecules adsorbed in a hexagon-down orientation. This means that the current in Eq. (16) must have $a_{x}=a_{y}$. Fig. 7 shows simulated constant current images for four values of $a_{z}$ between 0.35 and 0.65 (and taking $a_{x}+a_{y}+a_{z}=1$ ). We have chosen the current and background cutoff values such that the features in our simulated images are approximately the same size as the features in the experimental images. Comparison with the experimental images suggests that the dimmer images arise from a current with $a_{z} \approx 0.45$. For smaller values of $a_{z}$, too much of the electron density transfers from the center to the three lobes, and for larger values the image becomes too rounded. The brighter image probably has $a_{z}$ nearer to 0.65 , although the resolution of the experimental images is insufficient to make a more precise identification.

Again, we can get the required current in the absence of any JT effects. The current obtained by imaging two degenerate states with $\delta_{3}^{\prime}>0$ is

$$
I=\frac{1}{3}\left[(1-X)\left(\psi_{x}^{2}+\psi_{y}^{2}\right)+(1+2 X) \psi_{z}^{2}\right]
$$


where

$$
X=\frac{\Delta_{1}}{\sqrt{\delta_{3}^{\prime 2}+4 \Delta_{1}^{2}}} .
$$

When $\delta_{3}^{\prime}=0.6$, this suggests that $\Delta_{1}>0$, with likely values of $\Delta_{1} \approx 0.1$ for the dimmer images and 0.9 for the brighter images. However, as $a_{z}=0.65$ is close to its asymptotic limit of $2 / 3$ (when $\Delta_{1} \rightarrow \infty$ ), small changes in the required value for $a_{z}$ result in large changes in the corresponding value of $\Delta_{1}$ in this case.

When JT effects are included, we find we can get matches to the required form for the current for all allowed values of the quadratic coupling constants, as long as $\Delta_{1}$ is positive to ensure a sufficiently large contribution from $\psi_{z}^{2}$ in the expression for the current [60]. This includes cases where the JT effect in the absence of a surface interaction would give $D_{2 h}$ wells and those where it would give $C_{2 h}$ wells.

\section{Summary}

We have studied the combined effects of JT interactions, Coulomb (electronelectron) repulsion and interactions with a surface substrate on multiply-charged $\mathrm{C}_{60}$ anions. JT and Coulomb interactions result in a set of equivalent minima in the APES. The molecule will dynamically pseudorotate between these distortions, hindered by barriers in the APES caused by quadratic JT coupling. We have shown that the surface interaction removes some of the equivalencies between the minima. For some orientations and surface interaction strengths, the result is a single minimum and hence there will be a static distortion of the molecule. However, in other cases there will still be more than one minimum. This means that a dynamic JT effect will operate, albeit between a reduced subset of minima compared to when surface interactions are neglected.

Observed STM images will be a superposition of images due to all equivalent minimum-energy configurations. As a consequence, all images are predicted to contain contributions from the squares of the three single-electron $T_{1 u}$ states, with no contributions from cross-terms between different states. The key conclusion of our study is that all of the observed images of $\mathrm{C}_{60}^{3-}$ and $\mathrm{C}_{60}^{4-}$ ions in 
Ref. 16] can be explained with a model involving dynamic JT distortions combined with surface interactions and the Coulomb term splitting. Furthermore, we have used our matches for the different charge states to deduce relationships between the quadratic coupling constants and the strength of the surface interaction. We find that matches to the observed images of $\mathrm{C}_{60}^{4-}$ can be obtained for quadratic coupling constants that would give wells of $D_{5 d}$ or $D_{3 d}$ symmetry in the absence of surface interactions. This is consistent with information deduced from other sources (as discussed in the Introduction), and is also the most likely situation to occur according to analysis of the JT Hamiltonian [14], but differs from the interpretation in Ref. [16] which was of an intrinsically static JT distortion to $D_{2 h}$ symmetry.

It should be noted that our model does not take into account interactions between molecules. It is clear from the ordering of images from different molecules that interactions with neighboring ions are present. These could alter the shapes of the observed features. However, as there is very little difference between images of different molecules and they can't all interact with each other in the same way, it seems reasonable to assume that these effects will be small.

\section{Acknowledgments}

AJL gratefully acknowledges the support of EPSRC (UK) for funding this work through a Doctoral Training Account.

\section{References}

[1] S. Margadonna, K. Prassides, Recent advances in fullerene superconductivity, J. Solid State Chem. 168 (2) (2002) 639-652. doi:10.1006/jssc.2002.9762.

[2] Y. Iwasa, T. Takenobu, Superconductivity, Mott-Hubbard states, and molecular orbital order in intercalated fullerides, J. Phys.-Condens. Matter 15 (2003) R495-R519. doi:10.1088/0953-8984/15/13/202. 
[3] G. Klupp, K. Kamarás, N. M. Nemes, C. M. Brown, J. Leão, Static and dynamic Jahn-Teller effect in the alkali metal fulleride salts $\mathrm{A}_{4} \mathrm{C}_{60} \quad \mathrm{~A}=\mathrm{K}, \mathrm{Rb}, \mathrm{Cs}$, Phys. Rev. B 73 (2006) 085415. doi:10.1103/PhysRevB.73.085415

[4] G. Klupp, P. Matus, K. Kamarás, A. Y. Ganin, A. McLennan, M. J. Rosseinsky, Y. Takabayashi, M. T. McDonald, K. Prassides, Dynamic JahnTeller effect in the parent insulating state of the molecular superconductor $\mathrm{Cs}_{3} \mathrm{C}_{60}$, Nat. Commun. 3 (2012) 912. doi:10.1038/ncomms1910.

[5] A. L. Sobolewski, The cooperative dynamical Jahn-Teller effect in alkali doped fullerides, Chem. Phys. Lett. 267 (1997) 452-459. doi:10.1016/s0009-2614(97)00123-1.

[6] V. M. Loktev, E. A. Pashitskii, R. Shekhter, M. Jonson, Key role of intramolecular Jahn-Teller vibrations and multivalley nature of the band spectrum in the mechanism of superconductivity in doped $\mathrm{C}_{60}$ fullerites, Low Temperature Physics 28 (2002) 821-829. doi:10.1063/1.1528573.

[7] K. P. Sinha, A. Jindal, On the mechanism of room temperature superconductivity in substitutionally doped graphene, Solid State Commun. 180 (2014) 44-45. doi:10.1016/j.ssc.2013.11.009.

[8] M. Fabrizio, E. Tosatti, Nonmagnetic molecular Jahn-Teller Mott insulators, Phys. Rev. B 55 (20) (1997) 13465-13472. doi:10.1103/PhysRevB.55.13465.

[9] A. Auerbach, N. Manini, E. Tosatti, Electron-vibron interactions in charged fullerenes. I. Berry phases, Phys. Rev. B 49 (1994) 12998. doi:10.1103/PhysRevB.49.12998.

[10] J. L. Dunn, I. D. Hands, C. A. Bates, Pseudorotation in fullerene anions, J. Mol. Struct. 838 (2007) 60-65. doi:10.1016/j.molstruc.2006.12.066. 
[11] I. D. Hands, J. L. Dunn, C. A. Bates, Theoretical calculation of pseudorotation rates applicable to the $\mathrm{C}_{60}^{-}$ion, Phys. Rev. B 73 (2006) 235425. doi:10.1103/PhysRevB.77.115445,

[12] A. Ceulemans, L. G. Vanquickenborne, The epikernel principle, Structure and Bonding 71 (1989) 125.

[13] L. M. Sindi, I. D. Hands, J. L. Dunn, C. A. Bates, A Study of the Quadratic $p^{2} \otimes h$ Jahn-Teller System, J. Mol. Struct. 838 (2007) 78. doi:10.1016/j.molstruc.2007.01.035

[14] H. S. Alqannas, A. J. Lakin, J. A. Farrow, J. L. Dunn, Interplay between Coulomb and Jahn-Teller effects in icosahedral systems with triplet electronic states coupled to $h$-type vibrations, Phys. Rev. B 88 (2013) 165430. doi:10.1103/PhysRevB.88.165430.

[15] P. Dahlke, M. J. Rosseinsky, Molecular structure, orientational order, and Jahn-Teller distortion of the $\mathrm{C}_{60}^{4-}$ anion in $\mathrm{Cs}_{4} \mathrm{C}_{60}$, Chem. Mater. 14 (3) (2002) 1285-1291. doi:10.1021/cm010386s

[16] A. Wachowiak, R. Yamachika, K. H. Khoo, Y. Wang, M. Grobis, D. H. Lee, S. G. Louie, M. F. Crommie, Visualization of the Molecular JahnTeller Effect in an Insulating $\mathrm{K}_{4} \mathrm{C}_{60}$ Monolayer, Science 310 (5747) (2005) 468. doi:10.1126/science.1117303.

[17] C. Faber, J. L. Janssen, M. Côté, E. Runge, X. Blase, Electron-phonon coupling in the $\mathrm{C}_{60}$ fullerene within the many-body $\mathrm{GW}$ approach, Phys. Rev. B 84 (15) (2011) 155104. doi:10.1103/PhysRevB.84.155104.

[18] I. D. Hands, J. L. Dunn, C. A. Bates, M. J. Hope, S. R. Meech, D. L. Andrews, Vibronic interactions in the visible and nearinfrared spectra of $\mathrm{C}_{60}^{-}$anions, Phys. Rev. B 77 (2008) 115445. doi:10.1103/PhysRevB.77.115445

[19] S. Tomita, J. U. Andersen, E. Bonderup, P. Hvelplund, B. Liu, S. B. Nielsen, U. V. Pedersen, J. Rangama, K. Hansen, O. Echt, Dy- 
namic Jahn-Teller Effects in Isolated $\mathrm{C}_{60}^{-}$Studied by Near-Infrared Spectroscopy in a Storage Ring, Phys. Rev. Lett. 94 (5) (2005) 053002. doi:10.1103/PhysRevLett.94.053002

[20] K. Tanaka, M. Okada, K. Okahara, T. Yamabe, Structure and Electronic State of $\mathrm{C}_{60}$, Chem. Phys. Lett. 193 (1-3) (1992) 101-103. doi:10.1016/0009-2614(92)85690-c.

[21] L.-F. Yuan, J. Yang, H. Wang, C. Zeng, Q. Li, B. Wang, J. G. Hou, Q. Zhu, D. M. Chen, Low-temperature orientationally ordered structures of two-dimensional $\mathrm{C}_{60}$, J. Am. Chem. Soc. 125 (2003) 169-172. doi:10.1021/ja025775h.

[22] J. L. Dunn, A. J. Lakin, I. D. Hands, Manifestation of dynamic Jahn-Teller distortions and surface interactions in scanning tunnelling microscopy images of the fullerene anion $\mathrm{C}_{60}^{-}$, New J. Phys. 14 (8) (2012) 083038. doi:10.1088/1367-2630/14/8/083038

[23] N. Koga, K. Morokuma, Abinitio Mo Study of the $\mathrm{C}_{60}$ Anion Radical - the Jahn-Teller Distortion and Electronic-Structure, Chem. Phys. Lett. 196 (12) (1992) 191-196. doi:10.1016/0009-2614(92)85952-7.

[24] H. Ramanantoanina, M. Gruden-Pavlovic, M. Zlatar, C. Daul, Density functional theory study of the multimode Jahn-Teller problem in the fullerene anion, Int. J. Quantum Chem. 113 (2012) 802-807. doi:10.1002/qua.24080.

[25] J. L. Dunn, C. A. Bates, Analysis of the $T_{1 u} \otimes h_{g}$ Jahn-Teller system as a model for $\mathrm{C}_{60}$ molecules, Phys. Rev. B 52 (1995) 5996. doi:10.1103/PhysRevB.52.5996.

[26] C. C. Chancey, M. C. M. O'Brien, The Jahn-Teller Effect in $\mathrm{C}_{60}$ and other Icosahedral Complexes, Princeton University Press, Princeton, 1997.

[27] M. C. M. O'Brien, Vibronic energies in $\mathrm{C}_{60}^{n-}$ and the Jahn-Teller effect, Phys. Rev. B 53 (1996) 3775. doi:10.1103/PhysRevB.53.3775. 
[28] I. D. Hands, J. L. Dunn, C. A. Bates, Visualisation of static JahnTeller effects in the fullerene anion $\mathrm{C}_{60}^{-}$, Phys. Rev. B 82 (2010) 155425. doi:10.1103/PhysRevB.82.155425,

[29] Y. Wang, R. Yamachika, A. Wachowiak, M. Grobis, K. Khoo, D. H. Lee, S. G. Louie, M. F. Crommie, Novel Orientational Ordering and Reentrant Metallicity in $\mathrm{K}_{x} \mathrm{C}_{60}$ Monolayers for $3 \leq x \leq 5$, Phys. Rev. Lett. 99 (8) (2007) 086402. doi:10.1103/PhysRevLett.99.086402.

[30] K. Y. Amsharov, Y. Kramer, M. Jansen, Direct Observation of the Transition from Static to Dynamic Jahn-Teller Effects in the [Cs(THF)4]C 60 Fulleride, Angew. Chem.-Int. Edit. 50 (49) (2011) 1164011643. doi:10.1002/anie.201105360.

[31] N. Iwahara, T. Sato, K. Tanaka, L. F. Chibotaru, Vibronic coupling in $\mathrm{C}_{60}^{-}$anion revisited: Derivations from photoelectron spectra and DFT calculations, Phys. Rev. B 82 (24) (2010) 245409. doi:10.1103/PhysRevB.82.245409.

[32] R. E. Walstedt, D. W. Murphy, M. Rosseinsky, Structural Distortion in $\mathrm{Rb}_{3} \mathrm{C}_{60}$ Revealed By ${ }^{87} \mathrm{Rb}$ NMR, Nature 362 (6421) (1993) 611-613. doi:10.1038/362611a0

[33] A. J. Lakin, I. D. Hands, C. A. Bates, J. L. Dunn, The quadratic $p^{3} \otimes h$ Jahn-Teller system as a model for the $\mathrm{C}_{60}^{3-}$ anion, in: M. Atanasov, C. Daul (Eds.), Vibronic Interactions and the Jahn-Teller effect: Theory and Applications, Vol. 23 of Progress in Theoretical Chemistry and Physics, Springer, Dordrecht, 2012, pp. 231-243. doi:10.1007/978-94-007-2384-9_12.

[34] Y. Wang, R. Yamachika, A. Wachowiak, M. Grobis, M. F. Crommie, Tuning fulleride electronic structure and molecular ordering via variable layer index, Nature Mater. 7 (2008) 194. doi:10.1038/nmat2100.

965 [35] F. Schulz, M. Ijäs, R. Drost, S. K. Hämäläinen, A. Harju, A. P. Seitsonen, P. Liljeroth, Many-body transitions in a single molecule visual- 
ized by scanning tunnelling microscopy, Nat. Phys. 11 (2015) 229-234. doi:10.1038/nphys3212.

[36] P. W. Fowler, A. Ceulemans, Symmetry-relations in the property surfaces of icosahedral molecules, Mol. Phys. 54 (1985) 767. doi:10.1080/00268978500103151.

[37] S. Sookhun, J. L. Dunn, C. A. Bates, Jahn-Teller effects in the fullerene anion $\mathrm{C}_{60}^{2-}$, Phys. Rev. B 68 (2003) 235403. doi:10.1080/00268978500103151.

[38] M. C. M. O'Brien, Dynamic Jahn-Teller effect with many frequencies - a simple approach to a complicated problem, J. Phys. C: Solid State Phys. 5 (1972) 2045-2063. doi:10.1088/0022-3719/5/15/019.

[39] O. Gunnarsson, H. Handschuh, P. S. Bechthold, B. Kessler, G. Ganteför, W. Eberhardt, Photoemission Spectra of $\mathrm{C}_{60}^{-}$: Electron-Phonon Coupling, Jahn-Teller Effect, and Superconductivity in the Fullerides, Phys. Rev. Lett. 74 (1995) 1875. doi:10.1103/PhysRevLett.74.1875.

[40] X.-B. Wang, H.-K. Woo, L.-S. Wang, Vibrational cooling in a cold ion trap: Vibrationally resolved photoelectron spectroscopy of cold $\mathrm{C}_{60}^{-}$anions, J. Chem. Phys. 123 (2005) 051106. doi:10.1063/1.1998787.

[41] C. M. Varma, J. Zaanen, K. Raghavachari, Superconductivity in the fullerenes, Science $254 \quad$ (1991) 989-992. doi:10.1126/science.254.5034.989

[42] N. Breda, R. A. Broglia, G. Colò, H. E. Roman, F. Alasia, G. Onida, V. Ponomarev, E. Vigezzi, Electron-phonon coupling in charged buckminsterfullerene, Chem. Phys. Lett. 286 (3-4) (1998) 350-354. doi:10.1016/S0009-2614(98)00131-6

[43] N. Manini, A. Dal Corso, M. Fabrizio, E. Tosatti, Electron-vibration coupling constants in positively charged fullerene, Philos. Mag. B 81 (2001) 793. doi:10.1080/13642810108216543. 
[44] M. Saito, Electron-phonon coupling of electron- or hole-injected $\mathrm{C}_{60}$, Phys. Rev. B 65 (2002) 220508. doi:10.1103/PhysRevB.65.220508.

[45] T. Frederiksen, K. J. Franke, A. Arnau, G. Schulze, J. I. Pascual, N. Lorente, Dynamic Jahn-Teller effect in electron transport through single $\mathrm{C}_{60}$ molecules, Phys. Rev. B 78 (2008) 233401. doi:10.1103/PhysRevB.78.233401.

[46] J. Laflamme Janssen, M. Côté, S. G. Louie, M. L. Cohen, Electron-phonon coupling in $\mathrm{C}_{60}$ using hybrid functionals, Phys. Rev. B 81 (7) (2010) 073106. doi:10.1103/PhysRevB.81.073106

[47] N. Iwahara, L. F. Chibotaru, Dynamical Jahn-Teller Effect and Antiferromagnetism in $\mathrm{Cs}_{3} \mathrm{C}_{60}$, Phys. Rev. Lett. 111 (2013) 056401. doi:10.1103/PhysRevLett.111.056401.

[48] N. Iwahara, L. F. Chibotaru, Dynamical Jahn-Teller instability in metallic fullerides, Phys. Rev. B 91 (2015) 035109. doi:10.1103/PhysRevB.91.035109.

[49] K. Kundu, D. R. Kattnig, B. Mladenova, G. Grampp, R. Das, Electron Spin Relaxation of $\mathrm{C}_{60}$ Monoanion in Liquid Solution: Applicability of Kivelson-Orbach Mechanism, J. Phys. Chem. A 119 (2015) 3200. doi:10.1021/jp5126409.

[50] A. V. Nikolaev, B. N. Plakhutin, $\mathrm{C}_{60}$ fullerene as a pseudoatom of the icosahedral symmetry, Russ. Chem. Rev. 79 (9) (2010) 729-755. doi:10.1070/RC2010v079n09ABEH004139.

[51] A. V. Nikolaev, K. H. Michel, Molecular terms, magnetic moments, and optical transitions of molecular ions $\mathrm{C}_{60}^{m \pm}$, J. Chem. Phys. 117 (2002) 4761. doi:10.1063/1.1497644.

[52] R. L. Martin, J. P. Ritchie, Coulomb and exchange interactions in $\mathrm{C}_{60}^{n-}$, Phys. Rev. B 48 (7) (1993) 4845-4849. doi:10.1103/PhysRevB.48.4845. 
[53] I. D. Hands, J. L. Dunn, C. A. Bates, Calculation of images of oriented $\mathrm{C}_{60}$ molecules using molecular orbital theory, Phys. Rev. B 81 (2010) 205440. doi:10.1103/PhysRevB.81.205440

[54] A. J. Lakin, C. Chiutu, A. M. Sweetman, P. Moriarty, J. L. Dunn, Recovering molecular orientation from convoluted orbitals, Phys. Rev. B 88 (2013) 035447. doi:10.1103/PhysRevB.88.035447.

[55] A. J. Lakin, H. S. Alqannas, J. L. Dunn, Theoretical modelling of JahnTeller distorted $\mathrm{C}_{60}$ anions on a surface, J. Phys: Conference Series 428 (2013) 012001. doi:10.1088/1742-6596/428/1/012001.

[56] I. D. Hands, W. A. Diery, J. L. Dunn, C. A. Bates, The JahnTeller Effect in $\left(h_{u}^{+}\right)^{2} \otimes h_{g}$ Systems, J. Mol. Struct. 838 (2007) 66-73. doi:10.1016/j.molstruc.2006.12.060.

[57] I. D. Hands, W. A. Diery, C. A. Bates, J. L. Dunn, Jahn-Teller effects in the $\mathrm{C}_{60}^{2+}$ cation undergoing $D_{2 h}$ distortion, Phys. Rev. B 76 (2007) 085426. doi:10.1103/PhysRevB.76.085426.

[58] J. Tersoff, D. R. Hamann, Theory of the Scanning Tunneling Microscope, Phys. Rev. B 31 (1985) 805. doi:10.1103/PhysRevB.31.805.

[59] J. Bardeen, Tunnelling from a many-particle point of view, Phys. Rev. Lett. 6 (1961) 57. doi:10.1103/PhysRevLett.6.57.

[60] H. S. Alqannas, The Jahn-Teller and surface interactions in $\mathrm{C}_{60}$ nano systems, Ph.D. thesis, University of Nottingham, Nottingham, UK (2014).

[61] W. W. Pai, C.-L. Hsu, M. C. Lin, K. C. Lin, T. B. Tang, Structural relaxation of adlayers in the presence of adsorbate-induced reconstruction: $\quad \mathrm{C}_{60} / \mathrm{Cu}(111)$, Phys. Rev. $\mathrm{B} \quad 69$ (2004) 125405. doi:10.1103/PhysRevB.69.125405 